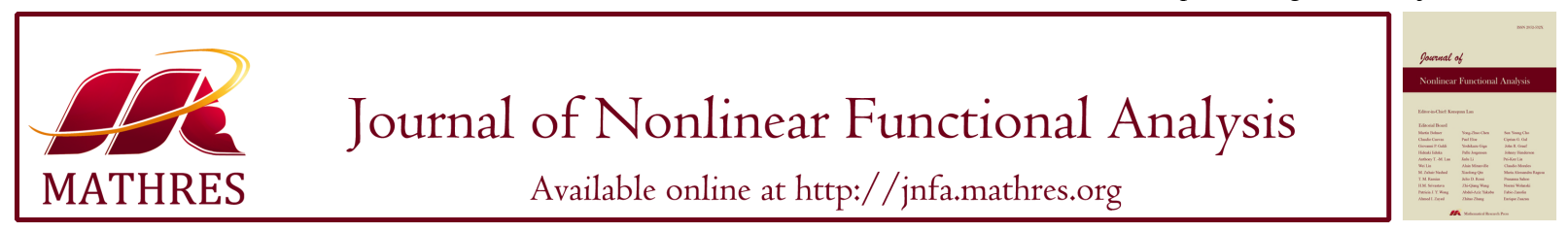

\title{
ROBUST OPTIMALITY AND DUALITY FOR MINIMAX FRACTIONAL PROGRAMMING PROBLEMS WITH SUPPORT FUNCTIONS
}

\author{
INDIRA P. DEBNATH ${ }^{1}, \mathrm{X} . \mathrm{QIN}^{2, *}$ \\ ${ }^{1}$ Department of Applied Mathematics and Humanities, \\ S. V. National Institute of Technology Surat, Gujarat-395007, India \\ ${ }^{2}$ National Yunlin University of Science and Technology, Douliou 64002, Taiwan
}

\begin{abstract}
In this paper, we consider a class of robust nondifferentiable minimax fractional programming problems containing support functions in both the objective functions and in the constraints. Using the robust subdifferentiable constraint qualification, we obtain necessary and sufficient optimality conditions for the robust convexconcave nondifferentiable minimax fractional problem. We introduce two types of robust dual problems, robust Wolfe dual and robust Mond-Weir dual. Moreover, we discuss the scenario uncertainty of a quadratic minimax fractional programming.
\end{abstract}

Keywords. Minimax fractional problem; Robust uncertainty; Support functions; Optimality and duality; Uncertain quadratic.

\section{INTRODUCTION}

In decision science, the optimization problems where both the minimization and the maximization processes are performed in the objectives are called minimax fractional programming problems. This type of problems has numerous applications in game theory [1], economics [2, 3], minimum risk problems [4], multiobjective programming [5], etc. Several researchers have investigated the optimality conditions/duality results for the generalized minimax fractional problems. Antczak [6] obtained the optimality conditions for a class of generalized minimax problems under invexity assumptions. Under generalized convexity assumptions, Ahmad and Husain [7] derived optimality conditions and the duality in nondifferentiable minimax fractional programming. Later, the second order duality for a nondifferentiable minimax fractional problem was discussed in Gupta, Dangar and Ahamd [8] under type I- functions. Fore more details, the readers are referred to $[9,10]$.

In several real world problems, the data involved in the optimization problems seem to be uncertain due to lack of information or error in measurement or estimation. To deal with such type

${ }^{*}$ Corresponding author.

E-mail addresses: idmath26@gmail.com (I.P. Debnath), qinx1@yuntech.edu.tw (X. Qin).

Received October 16, 2020; Accepted January 2, 2021.

(C)2021 Journal of Nonlinear Functional Analysis 
of situations, the robust optimization approach emerged as an efficient deterministic tool for studying optimization problems involving uncertainty in the data. Therefore, one can study robust optimization via its counterpart. Indeed, in robust optimization, no probability distribution information on the uncertain parameters are considered. Moreover, the way the robust solutions performs are being judged by the multiple objectives conflicting in nature, like, quality/profit against cost of the material. Henceforth, it is quite interesting to undergo deep investigation how the theory and applications of robust optimization problems in the practical world works. Doolittle, Kerivin and Wiecek [11] studied some theoretical techniques for multiobjective robust optimization problem and gave a relationship between the uncertain robust problem and its robust counterpart by considering some column-wise and row-wise uncertainty. With the developed approach, they gave an application to internet routing. An overview of robust uncertainty approach together with some algorithms to obtain robust optimal solutions was given in [12]. We refer to $[13,14,15]$ and the references therein.

In scalar as well as multiobjective optimization, we focus on finding global optimal solutions or global efficient solutions for the problem. But, in general, it is not always possible to obtain global optimal solutions, particularly, when it comes to the fact that these solutions are quite sensitive to even small perturbations of variables. For a nondifferentiable multiobjective robust optimization problem, Gunawan and Azarm [16] discussed the robust Pareto solution by using a sensitive region concept. Deb and Gupta [15] gave the difference between global and robust multiobjective optimization principles by discussing two different procedures to find the robust solutions for multiobjective robust optimization problems. Kuroiwa and Lee [17] presented three different kinds of finding robust efficient solutions for a multiobjective optimization problem with uncertainty. Gorisson [18] discussed the robust optimal solutions for the uncertain fractional optimization problems. Recently, Lee and Jiao [19] discussed how to find the efficient solutions of a multiobjective robust optimization problem by using the $\varepsilon$-constraint approach, which is a scalarization approach. Utilizing the same technique and an affinely parameterized uncertain data set, Jiao and Lee [20] gave the idea to obtain the efficient solutions of a multiple objectives as SOS-convex polynomials functions.

In optimization theory, it is very important to discuss the necessary and sufficient optimality conditions and duality. However, in literature, only a few papers are available which discussed the sufficient optimality conditions for fractional optimization problems with uncertainty both in the objectives and the constraints. For a robust fractional problem with constraint data uncertainty, Jeyakumar and $\mathrm{Li}$ [21] discussed the robust duality with the aid of a Slater-type constraint qualification. On the other hand, introducing a robust cone constraint qualification, Sun and Chai [22] established the robust strong duality for a fractional problem with uncertain data and also discussed its Wolfe dual. Goberna et al. [23] gave a formula which guarantees the constraints feasibility for all possible uncertainties under affine data parametrization. They also presented a characterization of robust weakly efficient solutions against rank one objective data uncertainty in matrix form. Chuong [24] established the necessary and sufficient optimality conditions for a robust multiobjective nonsmooth optimization problem in terms of multipliers and subdifferentials of the functions involved in the problem. Moreover, he also derived the duality relations between a robust dual and the considered problem under generalized convexity. In a recent work, Lee and Lee [25] considered a robust semi-infinite multiobjective problem and presented the optimality conditions for a weakly robust efficient solution for the problem. 
Moreover, an Wolfe dual to the problem is intoduced and some duality results are also discussed. In a recent work, Bokrantz and Fredriksson [26] provided optimality conditions for a robust optimization problem which states that the solution is efficient only when it is an optimal solution to a strictly increasing scalarized function. However, the fractional optimization problems in data uncertainty both in the numerator and the denominator of the objective functions together with the constraints has not been explored much in the literature. Using the concept of the subdifferentiability, Sun et al. [27] considered a robust fractional optimization problem with uncertain data and introduced robust subdifferentiable constraint qualification for the problem. With these constraint qualifications, some complete characterizations of the problem were given for robust optimal solutions. They have also extended the proposed approach to the multiobjective fractional problems with uncertain data. Recently, Li, Wang and Lin [28] studied the optimality conditions for a minimax robust nondifferentiable fractional programming problems by using the subdifferentiability constraint qualification introduced in [27]. Using these optimality conditions, they derived duality results for an Wolfe type and a Mond-Weir type dual problem.

To the best of our knowledge, there is no such paper which studied the minimax nondifferentiable fractional optimization problems involving support functions with data-uncertainty. Inspired and motivated by $[27,28]$, this paper is devoted to a class of minimax nondifferentiable fractional optimization problems under uncertainty and support functions in both the objective functions and in the constraints. The sectionwise description of the paper is as follows. In Section 2, we introduce the minimax nondifferentiable fractional optimization problem with support functions under uncertain data. We recall some known definitions and introduce nondifferentiable robust constraint qualifications for the minimax fractional problem with support functions. The main problem is then converted to its robust counterpart. Using a deterministic approach given in Dinkelbach [29], we, in Section 3, present the necessary and sufficient optimality conditions for the problem under the nondifferentiable robust subdifferentiable constraint qualification. In Section 4, two different types of robust dual problems are introduced- robust Wolfe dual and robust Mond-Weir dual. By converting the duals to their robust counterpart, we obtain weak and strong duality results. In Section 5, the last section, a particular form of the fractional programming problem, i.e., the quadratic fractional problem with uncertain data is considered. Using the scenario data unceratinty in quadratic fractional problem, the problem is converted to its robust counterpart and an Wolfe dual is given in its robust counterpart. Finally, a strong duality relation is established between the robust counterpart of quadratic minimax fractional problem and its Wolfe dual.

\section{PRELIMinaries}

In this section, some definitions and definitions are given which will be needed in the sequel. Let $R^{n}$ denote $n$-dimensional Euclidean space and let $R_{+}^{n}$ be its non-negative orthant. Let $K$ be a non-empty closed, convex cone in $R^{m}$ and let $\zeta_{+}^{n}=\left\{\left(\zeta_{i}\right) \in R_{+}^{n}: \sum_{i=1}^{n} \zeta_{i}=1\right\}$. For a non-empty set $E \subset R^{n}, \operatorname{co} E$ represents the convex hull of $E$.

Definition 2.1. Let $A$ be a non-empty subset in $R^{n}$. The indicator function $\delta_{A}: R^{n} \rightarrow R \cup\{+\infty\}$ is defined as

$$
\delta_{A}(x)= \begin{cases}0, & \text { if } x \in A \\ +\infty, & \text { if } x \notin A\end{cases}
$$


Definition 2.2. Let $C \subset R^{n}$ be a nonempty, closed and convex cone. The dual cone of $C$ is defined as

$$
C^{*}=\left\{x \in R^{n}:\left\langle x^{*}, x\right\rangle \geq 0, \forall x \in C\right\},
$$

which is also closed and convex.

Definition 2.3. [28] A vector valued mapping $f: R^{n} \rightarrow R^{m}$ is said to be $K$-convex if and only if, for any $u, v \in R^{n}$,

$$
f(\lambda u+(1-\lambda) v)-\lambda f(u)-(1-\lambda) f(v) \in-K .
$$

Therefore, a mapping $g$ is said to be $K$-concave if $-g$ is $K$-convex.

Definition 2.4. For a convex function $\phi: R^{n} \rightarrow R$, the subdifferential of $\phi$ at $x \in R^{n}$ is defined by

$$
\partial \phi(x)=\left\{x^{*} \in R^{n}: \phi(y)-\phi(x) \geq\left\langle x^{*}, y-x\right\rangle, \forall y \in R^{n}\right\} .
$$

Definition 2.5. A function $F: R^{n} \times R^{p} \rightarrow R$ is said to be a convex-concave function, i.e., $F(., p)$ is a convex function for any $p \in P \subset R^{p}$ and $F(x,$.$) is a concave function for any x \in X \subset R^{n}$.

Definition 2.6. A vector valued function $H: R^{n} \times R^{p} \rightarrow R^{m}$ is said to be a $K$-convex-concave function if $H(., r)$ is a $K$-convex function for any $r \in R \subset R^{p}$ and $H(x,$.$) is a K$-concave function for any $x \in X \subset R^{n}$.

Definition 2.7. [30] Let $\theta$ be a compact convex set in $R^{n}$. The support function of $\theta$ is defined by

$$
S(x \mid \theta)=\max \left\{x^{T} y: y \in \theta\right\} .
$$

The subdifferential of $S(x \mid \theta)$ is given by

$$
\partial S(x \mid \theta)=\left\{z \in \theta: z^{T} x=S(x \mid \theta)\right\} .
$$

Consider the nondifferentiable minimax fractional problem under data uncertainity:

$$
\text { (NUP) } \min _{x \in R^{n}} \max _{1 \leq i \leq k} \frac{F_{i}\left(x, p_{i}\right)+S\left(x \mid D_{i}\right)}{G_{i}\left(x, q_{i}\right)-S\left(x \mid E_{i}\right)}
$$

s.t.

$$
\begin{gathered}
\left\{H_{1}\left(x, r_{1}\right)+S\left(x \mid W_{1}\right), \ldots, H_{m}\left(x, r_{m}\right)+S\left(x \mid W_{m}\right)\right\} \in-K, \\
x \in S, i=1,2, \ldots, k,
\end{gathered}
$$

where $S$ is a non-empty, closed and convex set of $R^{n}$ and for, $i=1,2, \ldots, k, j=1,2, \ldots, m$,

(i) $F_{i}: R^{n} \times R^{p} \rightarrow R$ and $G_{i}: R^{n} \times R^{p} \rightarrow R$ are convex-concave on $R^{n} \times P_{i}$, and concaveconvex on $R^{n} \times Q_{i}$, respectively. $H=\left(H_{1}, H_{2}, \ldots, H_{m}\right): R^{n} \times R^{p} \rightarrow R^{m}$ is $K$-convexconcave on $R^{n} \times R_{j} . \quad p_{i} \in P_{i}, q_{i} \in Q_{i}$ and $r_{j} \in R_{j}$ are the parameters which indicates uncertainty in the objective functions and the constraints in (NUP). $P_{i} \subset R^{p}, Q_{i} \subset R^{p}$ and $R_{j} \subset R^{p}$, respectively, are the uncertain compact and convex sets.

(ii) $F_{i}\left(., p_{i}\right)+S\left(() \mid. D_{i}\right), p_{i} \in P_{i}$, are non-negative, and $G_{i}\left(., q_{i}\right)-S\left(() \mid. E_{i}\right), q_{i} \in Q_{i}$, are positive over the feasible set of (NUP), where $S\left(x \mid D_{i}\right), S\left(x \mid E_{i}\right)$ and $S\left(x \mid W_{j}\right)$ are the support functions such that the compact convex sets $D_{i} \subset R^{n}, E_{i} \subset R^{n}$ and $W_{j} \subset R^{n}$, respectively. 
Following the methodology given in [13] for a robust optimization problem, problem (NUP) can be converted to:

$$
\text { (SUP) } \min _{x \in R^{n}} \max _{1 \leq i \leq k} \max _{\left(p_{i}, q_{i}\right) \in P_{i} \times Q_{i}} \frac{F_{i}\left(x, p_{i}\right)+S\left(x \mid D_{i}\right)}{G_{i}\left(x, q_{i}\right)-S\left(x \mid E_{i}\right)}
$$

s.t.

$$
\begin{gathered}
\left\{H_{1}\left(x, r_{1}\right)+S\left(x \mid W_{1}\right), \ldots, H_{m}\left(x, r_{m}\right)+S\left(x \mid W_{m}\right)\right\} \in-K, \\
x \in S, r_{j} \in R_{j}, i=1,2, \ldots, k, \text { and } j=1,2, \ldots, m .
\end{gathered}
$$

The nondifferentiable robust feasible set for problem (SUP) is given as:

$$
C=\left\{x \in S:\left(H_{1}\left(x, r_{1}\right)+S\left(x \mid W_{1}\right), \ldots, H_{m}\left(x, r_{m}\right)+S\left(x \mid W_{m}\right)\right) \in-K, \forall r_{j} \in R_{j}\right\} .
$$

A solution is called a robust optimal solution for problem (NUP) if it is an optimal solution for problem (SUP).

Remark 2.8. If $S\left(x \mid D_{i}\right)=S\left(x \mid E_{i}\right)=S\left(x \mid W_{j}\right)$ vanishes $\forall i=1,2, \ldots, k, j=1,2, \ldots, m$ in problem (NUP), then the problem is reduced to the (UP) in [28].

Definition 2.9. The robust type constraint qualification holds at $x \in C$ iff

$$
\partial \delta_{C}(x)=\partial \delta_{S}(x)+\bigcup_{\substack{\mu \in K^{*}, r_{j} \in R_{j}, \sum_{j=1}^{m} \mu_{j}\left(H_{j}\left(x, r_{j}\right)+x^{T} w_{j}\right)=0}} \sum_{j=1}^{m} \mu_{j} \partial\left(H_{j}\left(., r_{j}\right)+S\left((.) \mid w_{j}\right)\right)(x) .
$$

That is, the robust type constraint qualification can be replaced by

$$
\partial \delta_{C}(x) \subset \partial \delta_{S}(x)+\bigcup_{\substack{\mu \in K^{*}, r_{j} \in R_{j}, \sum_{j=1}^{m} \mu_{j}\left(H_{j}\left(x, r_{j}\right)+x^{T} w_{j}\right)=0}} \sum_{j=1}^{m} \mu_{j} \partial\left(H_{j}\left(., r_{j}\right)+S\left((.) \mid W_{j}\right)\right)(x),
$$

since the other inclusion is easy to show.

Remark 2.10. Let $Y$ be a compact set in some metric space. Let $\left\{\zeta_{j}\right\}: R^{n} \rightarrow R, j \in Y$ be a collection of convex functions. Assume

$$
\zeta(x)=\sup \left\{\zeta_{j}(x)\right\}_{j \in Y}<+\infty, \forall x \in R^{n} .
$$

Then, $\zeta$ is a convex function [31].

Lemma 2.11. Let $\zeta($.$) be upper semicontinuous on Y$ for each $x \in R^{n}$. Then

$$
\partial \zeta(x)=\operatorname{co}\left\{\partial \zeta_{j}(x): j \in Y(x)\right\}
$$

where $Y(x):=\left\{j \in Y: \zeta_{j}(x)=\zeta(x)\right\}$ is the active index-set.

Due to the complex nature of the objective functions involved in (SUP) as fractions of functions, it is necessary to utilize a deterministic approach [29] to convert into a non-fractional problem (SUP) $\lambda$, where $\lambda>0$ is a parameter given below

$$
(\mathrm{SUP})_{\lambda} \min _{x \in R^{n}} \varphi(x, \lambda)
$$

$$
\begin{gathered}
\text { s.t. }\left(H_{1}\left(x, r_{1}\right)+S\left(x \mid W_{1}\right), \ldots, H_{m}\left(x, r_{m}\right)+S\left(x \mid W_{m}\right)\right) \in-K, \\
\forall w_{j} \in W_{j}, x \in S, j=1,2, \ldots, m,
\end{gathered}
$$


where $\varphi(x, \lambda)=\max \underset{\substack{1 \leq i \leq k \\\left(p_{i}, q_{i}\right) \in P_{i} \times Q_{i}}}{ }\left[\left(F_{i}\left(x, p_{i}\right)+S\left(x \mid D_{i}\right)\right)-\lambda\left(G_{i}\left(x, q_{i}\right)-S\left(x \mid E_{i}\right)\right)\right]$.

The following lemma gives a relation between the non-fractional problem (SUP) $\lambda$ and the problem (SUP).

Lemma 2.12. A feasible solution $x^{*}$ is a robust optimal solution of the problem (NUP) with value $\tilde{\lambda}$ iff $x^{*}$ is an optimal solution of the problem $(S U P)_{\lambda}$ with optimal value $\varphi\left(x^{*}, \tilde{\lambda}\right)=0$.

Proof. First, we assume that $x^{*} \in C$ is a robust optimal solution of the (NUP) with an optimal value $\tilde{\lambda}$. It follows that

$$
\max _{\substack{1 \leq i \leq k \\\left(p_{i}, q_{i}\right) \in P_{i} \times Q_{i}}} \frac{F_{i}\left(x, p_{i}\right)+S\left(x \mid D_{i}\right)}{G_{i}\left(x, q_{i}\right)-S\left(x \mid E_{i}\right)} \geq \max _{\substack{1 \leq i \leq k \\\left(p_{i}, q_{i}\right) \in P_{i} \times Q_{i}}} \frac{F_{i}\left(x^{*}, p_{i}\right)+S\left(x^{*} \mid D_{i}\right)}{G_{i}\left(x^{*}, q_{i}\right)-S\left(x^{*} \mid E_{i}\right)}=\tilde{\lambda} .
$$

Recall the fact that $F_{i}(x,$.$) is a concave and continuous function and G_{i}(x,$.$) is a convex and$ continuous function $i=1,2, \ldots, k$. Also, the compactness of $U_{i}$ and $V_{i}$ imply that, for some $i_{0}$, $p_{i_{0}} \in P_{i_{0}}$ and $q_{i_{0}} \in Q_{i_{0}}$,

$$
\begin{gathered}
\frac{F_{i_{0}}\left(x, p_{i_{0}}\right)+S\left(x \mid D_{i_{0}}\right)}{G_{i_{0}}\left(x, q_{i_{0}}\right)-S\left(x \mid E_{i_{0}}\right)} \geq \tilde{\lambda} \\
\Rightarrow\left[F_{i_{0}}\left(x, p_{i_{0}}\right)+S\left(x \mid D_{i_{0}}\right)\right]-\tilde{\lambda}\left[G_{i_{0}}\left(x, q_{i_{0}}\right)-S\left(x \mid E_{i_{0}}\right)\right] \geq 0 .
\end{gathered}
$$

Now, for $x \in C$, one has

$$
\begin{aligned}
\varphi(x, \tilde{\lambda})= & \max _{\substack{1 \leq i \leq k \\
\left(p_{i}, q_{i}\right) \in P_{i} \times Q_{i}}}\left[F_{i}\left(x, p_{i}\right)+S\left(x \mid D_{i}\right)\right]-\tilde{\lambda}\left[G_{i}\left(x, q_{i}\right)-S\left(x \mid E_{i}\right)\right] \\
\geq & {\left[F_{i_{0}}\left(x, p_{i_{0}}\right)+S\left(x \mid D_{i_{0}}\right)\right]-\tilde{\lambda}\left[G_{i_{0}}\left(x, q_{i_{0}}\right)-S\left(x \mid E_{i_{0}}\right)\right] }
\end{aligned}
$$

Therefore, using (2.1), we have

$$
\varphi(x, \tilde{\lambda}) \geq 0
$$

Again, the definition of $\tilde{\lambda}$ implies that

$$
\begin{gathered}
\frac{F_{i}\left(x^{*}, p_{i}\right)+S\left(x^{*} \mid D_{i}\right)}{G_{i}\left(x^{*}, q_{i}\right)-S\left(x^{*} \mid E_{i}\right)} \leq \tilde{\lambda}, i=1,2, \ldots, k \\
\Rightarrow\left\{F_{i}\left(x^{*}, p_{i}\right)+S\left(x^{*} \mid D_{i}\right)\right\}-\tilde{\lambda}\left\{G_{i}\left(x^{*}, q_{i}\right)-S\left(x^{*} \mid E_{i}\right)\right\} \leq 0, i=1,2, \ldots, k .
\end{gathered}
$$

This yields

$$
\max _{1 \leq i \leq k}\left[\left\{F_{i}\left(x^{*}, p_{i}\right)+S\left(x^{*} \mid D_{i}\right)\right\}-\tilde{\lambda}\left\{G_{i}\left(x^{*}, q_{i}\right)-S\left(x^{*} \mid E_{i}\right)\right\}\right] \leq 0
$$

which further implies

$$
\varphi\left(x^{*}, \tilde{\lambda}\right) \leq 0 .
$$

Equation (2.2) together with equation (2.3) implies $0=\varphi\left(x^{*}, \tilde{\lambda}\right) \leq \varphi(x, \tilde{\lambda})$. Hence $x^{*}$ is the optimal solution of the (SUP) $\lambda$ with optimal value $\varphi\left(x^{*}, \tilde{\lambda}\right)=0$. 
Conversely, let $x^{*}$ be an optimal solution of the (SUP) $\lambda$ with optimal value $\varphi\left(x^{*}, \tilde{\lambda}\right)=0$. This gives, for any $p_{i} \in P_{i}, q_{i} \in Q_{i}, i=1,2, \ldots, k$,

$$
\begin{aligned}
& \varphi(x, \tilde{\lambda}) \geq \varphi\left(x^{*}, \tilde{\lambda}\right)=0, \forall x \in S . \\
& \Rightarrow\left[\left\{F_{i}\left(x, p_{i}\right)+S\left(x \mid D_{i}\right)\right\}-\tilde{\lambda}\left\{G_{i}\left(x, q_{i}\right)-S\left(x \mid E_{i}\right)\right\}\right] \geq 0 \\
& \Rightarrow \frac{F_{i}\left(x, p_{i}\right)+S\left(x \mid D_{i}\right)}{G_{i}\left(x, q_{i}\right)-S\left(x \mid E_{i}\right)} \geq \tilde{\lambda}, 1 \leq i \leq k \\
& \Rightarrow \quad \max _{\substack{1 \leq i \leq k \\
\left(p_{i}, q_{i}\right) \in P_{i} \times Q_{i}}} \frac{F_{i}\left(x, p_{i}\right)+S\left(x \mid D_{i}\right)}{G_{i}\left(x, q_{i}\right)-S\left(x \mid E_{i}\right)} \geq \tilde{\lambda} .
\end{aligned}
$$

This further implies

$$
\max _{\substack{1 \leq i \leq k \\\left(p_{i}, q_{i}\right) \in P_{i} \times Q_{i}}} \frac{F_{i}\left(x, p_{i}\right)+S\left(x \mid D_{i}\right)}{G_{i}\left(x, q_{i}\right)-S\left(x \mid E_{i}\right)} \geq \tilde{\lambda}=\max _{\substack{1 \leq i \leq k \\\left(p_{i}, q_{i}\right) \in P_{i} \times Q_{i}}} \frac{F_{i}\left(x^{*}, p_{i}\right)+S\left(x^{*} \mid D_{i}\right)}{G_{i}\left(x^{*}, q_{i}\right)-S\left(x^{*} \mid E_{i}\right)} .
$$

Hence, $x^{*}$ is an optimal solution of (NUP).

\section{Optimality CONDitions Under UnCERTAinty}

In this section, we discuss the necessary and sufficient optimality conditions for (NUP) under robust subdifferential constraint qualifications with uncertainty parameters in the both the numerator and the denominator of the objective functions. Since (NUP) is a non-convex optimization problem, problem (SUP) can be converted to a deterministic non-fractional problem $(\mathrm{SUP})_{\lambda}$. by virtue of a deterministic approach used in Dinkelbach [29].

Theorem 3.1. Let $\tilde{x}$ be a feasible point of problem (NUP) and the robust constraint qualifications (RSCQ) hold at $\tilde{x}$. Then $\tilde{x}$ is an optimal solution for (NUP) having optimal value $\lambda$ iff there exist $\tilde{u}_{i} \in \zeta_{+}^{k}, \tilde{p}_{i} \in P_{i}, \tilde{q}_{i} \in Q_{i}, \tilde{r}_{j} \in R_{j}$ and $\tilde{\gamma}_{j} \in K^{*}, i=1,2, \ldots, k, j=1,2, \ldots$, m such that

$$
\begin{gathered}
0 \in \sum_{i=1}^{k} \tilde{u}_{i}\left[\left(\partial F_{i}\left(., \tilde{p}_{i}\right)(\tilde{x})+d_{i}\right)-\lambda\left(\partial G_{i}\left(., \tilde{q}_{i}\right)(\tilde{x})+e_{i}\right)\right]+\partial \delta_{C}(\tilde{x})+\sum_{j=1}^{m}\left[\tilde{\gamma}_{i}\left(\partial H_{j}\left(., \tilde{r}_{j}\right)+w_{j}\right)\right] \\
\sum_{i=1}^{k} \tilde{u}_{j}\left[\left(F_{i}\left(\tilde{x}, \tilde{p}_{i}\right)+\tilde{x}^{T} d_{i}\right)-\lambda\left(G_{i}\left(\tilde{x}, \tilde{q}_{i}\right)-\tilde{x}^{T} e_{i}\right)\right]=0 \\
\sum_{j=1}^{m} \tilde{\gamma}_{j}\left(H_{j}\left(\tilde{x}, \tilde{r}_{j}\right)+\tilde{x}^{T} w_{j}\right)=0 . \\
S\left(\tilde{x} \mid D_{i}\right)=\tilde{x}^{T} d_{i}, \\
S\left(\tilde{x} \mid E_{i}\right)=\tilde{x}^{T} e_{i}, \\
S\left(\tilde{x} \mid W_{j}\right)=\tilde{x}^{T} w_{j}, \\
\sum_{i=1}^{k} \tilde{u}_{i}=1, \tilde{u}_{i} \geq 0, d_{i} \in D_{i}, e_{i} \in E_{i}, w_{j} \in W_{j} .
\end{gathered}
$$


Proof. Suppose that $\tilde{x}$ is an optimal solution of (NUP). By Lemma 2.12, we have that $\tilde{x}$ is an optimal solution of (SUP) $\lambda_{\lambda}$. We know that the support functions are convex sets. Hence, $S\left(() \mid. D_{i}\right)$ and $S\left(() \mid. E_{i}\right)$ are convex. $F_{i}\left(., p_{i}\right),-G_{i}\left(., q_{i}\right) i=1,2, \ldots, k$ are also convex functions. This implies that $F_{i}\left(., p_{i}\right)+S\left(. \mid D_{i}\right)$ and $-G_{i}\left(., q_{i}\right)+S\left(. \mid E_{i}\right)$ are convex functions. Consequently, $\varphi((),. \lambda)$ is also convex and then there exists $\hat{x} \in \partial \varphi(., \lambda)(\tilde{x})$ such that $-\hat{x} \in \partial \delta(\tilde{x})$.

Further, $F_{i}\left(., p_{i}\right)+S\left(() \mid. D_{i}\right)$ and $-G_{i}\left(., q_{i}\right)+S\left(() \mid. E_{i}\right)$ are locally Lipschitz functions. Since $P_{i}$ and $Q_{i}$ are compact sets for each $i=1,2, \ldots, k$, we obtain from Lemma 2.11 that

$$
\partial \varphi(\tilde{x}, \lambda)=\operatorname{co}\left\{\left[\left(\partial F_{i}\left(., p_{i}\right)+d_{i}\right)-\lambda\left(\partial G_{i}\left(., q_{i}\right)-e_{i}\right)\right](\tilde{x}):\left(i, p_{i}, q_{i}\right) \in I(\tilde{x})\right\},
$$

where

$$
I(\tilde{x})=\left\{\left(i, p_{i}, q_{i}\right):\left(F_{i}\left(\tilde{x}, p_{i}\right)+\tilde{x}^{T} d_{i}\right)-\lambda\left(G_{i}\left(\tilde{x}, q_{i}\right)-\tilde{x}^{T} e_{i}\right)=\varphi(\tilde{x}, \lambda)=0\right\} .
$$

For some $l, n \in N, p_{i j} \in P_{i}, j=1,2, \ldots, l$ and $q_{i r} \in Q_{i}, r=1,2, \ldots, n$ such that

$$
\tilde{x}_{i j r}-d_{i}-\lambda e_{i} \in \partial\left[F_{i}\left(., p_{i j}\right)-\lambda G_{i}\left(., q_{i r}\right)\right](\tilde{x}), u_{i j r} \geq 0,
$$

where $\sum_{i=1}^{k} \sum_{j=1}^{l} \sum_{r=1}^{n} u_{i j r}=1$,

$$
\begin{gathered}
\hat{x}=\sum_{i=1}^{k} \sum_{j=1}^{l} \sum_{r=1}^{n} u_{i j r} \tilde{x}_{i j r} \text { and } \\
u_{i j r}\left[\left(F_{i}\left(\tilde{x}, p_{i j}\right)+\tilde{x}^{T} d_{i}\right)-\lambda\left(G_{i}\left(\tilde{x}, q_{i r}\right)-\tilde{x}^{T} e_{i}\right)\right]=0 .
\end{gathered}
$$

Setting $u_{i}=\sum_{j=1}^{l} \sum_{r=1}^{n} u_{i j r}$, we have $u_{i} \geq 0, \sum_{i=1}^{k} u_{i}=1$. For any $\hat{p}_{i} \in P_{i}$ and $\hat{q}_{i} \in Q_{i}$, we define

$$
\tilde{p}_{i}(x)= \begin{cases}\sum_{j=1}^{l} \sum_{r=1}^{n} \frac{u_{i j r}}{u_{i}} p_{i j}, & \text { if } u_{i}>0 \\ \hat{p}_{i}, & \text { if } u_{i}=0\end{cases}
$$

and

$$
\tilde{q}_{i}(x)= \begin{cases}\sum_{j=1}^{l} \sum_{r=1}^{n} \frac{u_{i j r}}{u_{i}} q_{i r}, & \text { if } q_{i}>0 \\ \hat{q}_{i}, & \text { if } u_{i}=0 .\end{cases}
$$

From the fact that $P_{i}$ and $Q_{i}$ are convex sets, we have $\tilde{p}_{i} \in P_{i}$ and $\tilde{q}_{i} \in Q_{i}$. Since $F_{i}(x,$.$) are$ concave and $S\left(x \mid D_{i}\right) \geq x^{T} d_{i}, d_{i} \in D_{i}, i=1,2, \ldots, k$, we have

$$
F_{i}\left(\sum_{j=1}^{l} \sum_{r=1}^{n} \frac{u_{i j r}}{u_{i}}\left(x, p_{i j}\right)\right)+S\left(x \mid D_{i}\right) \geq \sum_{j=1}^{l} \sum_{r=1}^{n} \frac{u_{i j r}}{u_{i}} F_{i}\left(x, p_{i j}\right)+x^{T} d_{i},
$$

which implies

$$
u_{i} F_{i}\left(x, \tilde{p}_{i}\right)+u_{i} S\left(x \mid D_{i}\right) \geq \sum_{j=1}^{l} \sum_{r=1}^{n} u_{i j r} F_{i}\left(x, p_{i j}\right)+\sum_{j=1}^{l} \sum_{r=1}^{n} u_{i j r} x^{T} d_{i} .
$$

Since $-G_{i}(x,$.$) are concave and S\left(x \mid E_{i}\right) \geq x^{T} e_{i}, e_{i} \in E_{i}, i=1,2, \ldots, k$, we get

$$
-G_{i}\left(\sum_{j=1}^{l} \sum_{r=1}^{n} \frac{u_{i j r}}{u_{i}}\left(x, q_{i r}\right)\right)+S\left(x \mid E_{i}\right) \geq \sum_{j=1}^{l} \sum_{r=1}^{n} \frac{u_{i j r}}{u_{i}}\left(-G_{i}\left(x, q_{i r}\right)\right)+x^{T} e_{i} .
$$


Since $\lambda>0$, the above expression implies

$$
-\lambda u_{i} G_{i}\left(x, \tilde{q}_{i}\right)+\lambda u_{i} S\left(x \mid E_{i}\right) \geq \sum_{j=1}^{l} \sum_{r=1}^{n} u_{i j r}\left(-\lambda G_{i}\left(x, q_{i r}\right)\right)+\lambda \sum_{j=1}^{l} \sum_{r=1}^{n} u_{i j r} x^{T} e_{i}
$$

Adding (3.7) and (3.8) and taking the summation over $i=1,2, \ldots, k$, we obtain

$$
\begin{aligned}
& \sum_{i=1}^{k} u_{i}\left[\left(F_{i}\left(x, \tilde{p}_{i}\right)+S\left(x \mid D_{i}\right)\right)-\lambda\left(G_{i}\left(x, \tilde{q}_{i}\right)-S\left(x \mid E_{i}\right)\right)\right] \\
& \geq \sum_{i=1}^{k} \sum_{j=1}^{l} \sum_{r=1}^{n} u_{i j r}\left[\left(F_{i}\left(x, p_{i j}\right)+x^{T} d_{i}\right)-\lambda\left(G_{i}\left(x, q_{i r}\right)-x^{T} e_{i}\right)\right] .
\end{aligned}
$$

From (3.4), we have

$$
\left[F_{i}\left(x, \tilde{p}_{i j}\right)-\lambda G_{i}\left(x, \tilde{q}_{i r}\right)\right]-\left[F_{i}\left(\tilde{x}, \tilde{p}_{i j}\right)-\lambda G_{i}\left(\tilde{x}, \tilde{q}_{i r}\right)\right] \geq\left\langle\left(\tilde{x}_{i j r}-d_{i}-\lambda e_{i}\right), x-\tilde{x}\right\rangle
$$

which yields

$$
\begin{aligned}
& {\left[\left(F_{i}\left(x, \tilde{p}_{i j}\right)+x^{T} d_{i}\right)-\lambda\left(G_{i}\left(x, \tilde{q}_{i r}\right)-x^{T} e_{i}\right)\right]-\left[\left(F_{i}\left(\tilde{x}, \tilde{p}_{i j}\right)+\tilde{x}^{T} d_{i}\right)-\lambda\left(G_{i}\left(\tilde{x}, \tilde{q}_{i r}\right)-\tilde{x}^{T} e_{i}\right)\right]} \\
& \geq\left\langle\tilde{x}_{i j r}, x-\tilde{x}\right\rangle .
\end{aligned}
$$

Also, we have from (3.6) that

$$
\sum_{i=1}^{k} \sum_{j=1}^{l} \sum_{r=1}^{n} u_{i j r}\left[\left(F_{i}\left(\tilde{x}, p_{i j}\right)+\tilde{x}^{T} d_{i}\right)-\lambda\left(G_{i}\left(\tilde{x}, q_{i r}\right)-\tilde{x}^{T} e_{i}\right)\right]=0
$$

Taking the summation over $i=1,2, \ldots, k$ in (3.10) and using (3.11), we have

$$
\sum_{i=1}^{k} \sum_{j=1}^{l} \sum_{r=1}^{n} u_{i j r}\left[\left(F_{i}\left(x, \tilde{p}_{i j}\right)+x^{T} d_{i}\right)-\lambda\left(G_{i}\left(x, \tilde{q}_{i r}\right)-x^{T} e_{i}\right)\right] \geq\left\langle\sum_{i=1}^{k} \sum_{j=1}^{l} \sum_{r=1}^{n} u_{i j r} \tilde{x}_{i j r}, x-\hat{x}\right\rangle .
$$

It follows from (3.5), (3.9) and (3.12) that

$$
\sum_{i=1}^{k} u_{i}\left[\left(F_{i}\left(x, \tilde{p}_{i}\right)+S\left(x \mid D_{i}\right)\right)-\lambda\left(G_{i}\left(x, \tilde{q}_{i}\right)-S\left(x \mid E_{i}\right)\right)\right] \geq\langle\hat{x}, x-\tilde{x}\rangle .
$$

Taking $x=\tilde{x}$ in (3.13), we get

$$
\sum_{i=1}^{k} u_{i}\left[\left(F_{i}\left(\tilde{x}, \tilde{p}_{i}\right)+\tilde{x}^{T} d_{i}\right)-\lambda\left(G_{i}\left(\tilde{x}, \tilde{q}_{i}\right)-\tilde{x}^{T} e_{i}\right)\right] \geq 0
$$

Since $\tilde{x}$ is an optimal solution of the (NUP) with optimal value $\lambda$, we find from (3.14) that

$$
\left.\sum_{i=1}^{k} u_{i}\left[\left(F_{i}\left(\tilde{x}, \tilde{p}_{i}\right)+S\left(\tilde{x} \mid D_{i}\right)\right)-\lambda\left(G_{i}\left(x, \tilde{q}_{i}\right)-S\left(\tilde{x} \mid E_{i}\right)\right)\right)\right]=0 .
$$

On the other hand, (3.13) and (3.15) imply that

$$
\hat{x} \in \partial \sum_{i=1}^{k} u_{i}\left[\left(F_{i}\left(., p_{i}\right)+S\left((.) \mid D_{i}\right)\right)-\lambda\left(G_{i}\left(., q_{i}\right)-S\left((.) \mid E_{i}\right)\right)\right](\tilde{x}),
$$


which yields that

$$
\hat{x} \in \sum_{i=1}^{k} u_{i} \partial\left[\left(F_{i}\left(., p_{i}\right)+S\left((.) \mid D_{i}\right)\right)-\lambda\left(G_{i}\left(., q_{i}\right)-S\left((.) \mid E_{i}\right)\right)\right](\tilde{x}) .
$$

Since $\left[\left(F_{i}\left(., p_{i}\right)+S\left(() \mid. D_{i}\right)\right)-\lambda\left(G_{i}\left(., q_{i}\right)-S\left(() \mid. E_{i}\right)\right)\right], i=1,2, \ldots, k$ is locally Lipschitz and continuous at $\tilde{x} \in R^{n}$, we have

$$
\begin{aligned}
& \partial\left[\left(F_{i}\left(., p_{i}\right)+S\left((.) \mid D_{i}\right)\right)-\lambda\left(G_{i}\left(., q_{i}\right)-S\left((.) \mid E_{i}\right)\right)\right](\tilde{x}) \\
& =\left(\partial F_{i}\left(., p_{i}\right)(\tilde{x})+d_{i}\right)-\lambda\left(\partial G_{i}\left(., q_{i}\right)(\tilde{x})-e_{i}\right) .
\end{aligned}
$$

Hence, (3.16) is equivalent to

$$
\hat{x} \in \sum_{i=1}^{k} u_{i}\left[\left(\partial F_{i}\left(., p_{i}\right)(\tilde{x})+d_{i}\right)-\lambda\left(\partial G_{i}\left(., q_{i}\right)(\tilde{x})-e_{i}\right)\right] .
$$

From the fact that $-\hat{x} \in \partial \delta_{S}(\tilde{x})[[21]$, Theorem 27.4] and (RSCQ), we get

$$
-\hat{x} \in \partial \delta_{C}(\tilde{x})+\underbrace{}_{\substack{\gamma \in K^{*}, w_{j} \in H_{j}, r_{j} \in R_{j} \\ \sum_{j=1}^{m} \gamma_{j}\left(H_{j}\left(\tilde{x}, r_{j}\right)+\tilde{x}^{T} w_{j}\right)=0}} \sum_{j=1}^{m} \gamma\left(\partial H_{j}\left(., r_{j}\right)(\tilde{x})+w_{j}\right) .
$$

Hence, there exist $\tilde{\gamma} \in K^{*}, \tilde{w}_{j} \in H_{j}$ and $\tilde{r}_{j} \in R_{j}$ such that

$$
-\hat{x} \in \partial \delta_{C}(\tilde{x})+\sum_{j=1}^{m} \tilde{\gamma}_{j}\left(\partial H_{j}\left(., \tilde{r}_{j}\right)(\tilde{x})+\tilde{w}_{j}\right)
$$

and

$$
\sum_{j=1}^{m} \tilde{\gamma}_{j}\left(H_{j}\left(\tilde{x}, \tilde{r}_{j}\right)+\tilde{x}^{T} \tilde{w}_{j}\right)=0 .
$$

Adding (3.17) and (3.18), we obtain

$$
\begin{aligned}
& 0 \in \sum_{i=1}^{k} u_{i}\left[\left(\partial F_{i}\left(., p_{i}\right)(\tilde{x})+d_{i}\right)-\lambda\left(\partial G_{i}\left(., q_{i}\right)(\tilde{x})-e_{i}\right)\right]+\delta_{C}(\tilde{x})+\sum_{j=1}^{m} \tilde{\gamma}_{j}\left(\partial H_{j}\left(., \tilde{r}_{j}\right)(\tilde{x})+\tilde{w}_{j}\right), \\
& \sum_{j=1}^{m} \tilde{\gamma}_{j}\left(H_{j}\left(\tilde{x}, \tilde{r}_{j}\right)+\tilde{x}^{T} \tilde{w}_{j}\right)=0 .
\end{aligned}
$$

Letting $u_{i}=\tilde{u}_{i} \in \zeta_{+}^{k}$, and using $S\left(\tilde{x} \mid D_{i}\right)=\tilde{x}^{T} d_{i}$ and $S\left(\tilde{x} \mid E_{i}\right)=\tilde{x}^{T} e_{i}$, together with (3.15) and (3.19), we obtain the expressions (3.1), (3.2) and (3.3).

Conversely, assume that $\exists \tilde{u}_{i} \in \zeta_{+}^{k}, \tilde{p}_{i} \in P_{i}, \tilde{q}_{i} \in Q_{i}, \tilde{r}_{j} \in R_{j}$ and $\tilde{\gamma}_{j} \in K^{*} \operatorname{such}$ that (3.1), (3.2) and (3.3) are satisfied. From (3.2), we have

$$
\begin{aligned}
0 & \in \sum_{i=1}^{k} \tilde{u}_{i}\left[\partial F_{i}\left(., \tilde{p}_{i}\right)(\tilde{x})-\lambda \partial G_{i}\left(., \tilde{q}_{i}\right)(\tilde{x})\right] \\
& +\partial \delta_{C}(\tilde{x})+\sum_{j=1}^{m} \tilde{\gamma}_{j} \partial H_{j}\left(., r_{j}\right)(\tilde{x})+\sum_{i=1}^{k} \tilde{u}_{i}\left(d_{i}+\lambda e_{i}\right)+\sum_{j=1}^{m} \tilde{\gamma}_{j} w_{j} .
\end{aligned}
$$


Hence, there exist

$$
\tilde{y}_{1} \in \sum_{i=1}^{k} \tilde{u}_{i}\left[\partial F_{i}\left(., \tilde{p}_{i}\right)(\tilde{x})-\lambda \partial G_{i}\left(., \tilde{q}_{i}\right)(\tilde{x})\right],
$$

$\tilde{y}_{2} \in \partial \delta_{C}(\tilde{x})$ and $\tilde{y}_{3} \in \sum_{j=1}^{m} \tilde{\gamma}_{j} \partial H_{j}\left(., r_{j}\right)(\tilde{x})$ such that

$$
\tilde{y}_{1}+\tilde{y}_{2}+\tilde{y}_{3}+\sum_{i=1}^{k} \tilde{u}_{i}\left(d_{i}+\lambda e_{i}\right)+\sum_{j=1}^{m} \tilde{\gamma}_{j} w_{j}=0 .
$$

Now, $\tilde{y}_{1} \in \sum_{i=1}^{k} \tilde{u}_{i}\left[\partial F_{i}\left(., \tilde{p}_{i}\right)(\tilde{x})-\lambda \partial G_{i}\left(., \tilde{q}_{i}\right)(\tilde{x})\right]$ implies

$$
\sum_{i=1}^{k} \tilde{u}_{i}\left[F_{i}\left(x, \tilde{p}_{i}\right)-\lambda G_{i}\left(x, \tilde{q}_{i}\right)\right]-\sum_{i=1}^{k} \tilde{u}_{i}\left[F_{i}\left(\tilde{x}, \tilde{p}_{i}\right)-\lambda G_{i}\left(\tilde{x}, \tilde{q}_{i}\right)\right] \geq\left\langle\tilde{y}_{1}, x-\tilde{x}\right\rangle .
$$

Again, $\tilde{y}_{2} \in \partial \delta_{C}(\tilde{x})$ gives

$$
0 \geq\left\langle\tilde{y}_{2}, x-\tilde{x}\right\rangle
$$

and $\tilde{y}_{3} \in \sum_{j=1}^{m} \tilde{\gamma}_{j} \partial H_{j}\left(., r_{j}\right)(\tilde{x})$ implies

$$
\sum_{j=1}^{m} \tilde{\gamma}_{j} H_{j}\left(x, r_{j}\right)-\sum_{j=1}^{m} \tilde{\gamma}_{j} H_{j}\left(\tilde{x}, r_{j}\right) \geq\left\langle\tilde{y}_{3}, x-\tilde{x}\right\rangle .
$$

Summing (3.21)-(3.23), we obtain

$$
\begin{aligned}
& \sum_{i=1}^{k} \tilde{u}_{i}\left[F_{i}\left(x, \tilde{p}_{i}\right)-\lambda G_{i}\left(x, \tilde{q}_{i}\right)\right]-\sum_{i=1}^{k} \tilde{u}_{i}\left[F_{i}\left(\tilde{x}, \tilde{p}_{i}\right)-\lambda G_{i}\left(\tilde{x}, \tilde{q}_{i}\right)\right]+\sum_{j=1}^{m} \tilde{\gamma}_{j} H_{j}\left(x, r_{j}\right)-\sum_{j=1}^{m} \tilde{\gamma}_{j} H_{j}\left(\tilde{x}, r_{j}\right) \\
& \geq\left\langle\tilde{y}_{1}+\tilde{y}_{2}+\tilde{y}_{3}, x-\tilde{x}\right\rangle .
\end{aligned}
$$

Using equation (3.20), we arrive at

$$
\sum_{i=1}^{k} \tilde{u}_{i}\left[F_{i}\left(x, \tilde{p}_{i}\right)-\lambda G_{i}\left(x, \tilde{q}_{i}\right)\right]+\sum_{j=1}^{m} \tilde{\gamma}_{j} H_{j}\left(x, r_{j}\right) \geq-\sum_{i=1}^{k} \tilde{u}_{i}\left(x^{T} d_{i}+\lambda x^{T} e_{i}\right)-\sum_{j=1}^{m} \tilde{\gamma}_{j} x^{T} w_{j},
$$

which implies

$$
\sum_{i=1}^{k} \tilde{u}_{i}\left[\left(F_{i}\left(x, \tilde{p}_{i}\right)+x^{T} d_{i}\right)-\lambda\left(G_{i}\left(x, \tilde{q}_{i}\right)-x^{T} e_{i}\right)\right] \geq-\sum_{j=1}^{m} \tilde{\gamma}_{j}\left(H_{j}\left(x, \tilde{r}_{j}\right)+x^{T} w_{j}\right) .
$$

Using $S\left(x \mid W_{j}\right) \geq x^{T} w_{j}, \tilde{\gamma}_{j} \in K^{*}$ and $-\left\{H_{j}\left(x, \tilde{r}_{j}\right)+S\left(x \mid W_{j}\right)\right\} \in K$ in (3.24), we get

$$
\sum_{i=1}^{k} \tilde{u}_{i}\left[\left(F_{i}\left(x, \tilde{p}_{i}\right)+x^{T} d_{i}\right)-\lambda\left(G_{i}\left(x, \tilde{q}_{i}\right)-x^{T} e_{i}\right)\right] \geq 0
$$

Since

$$
F_{i}\left(x, \tilde{p}_{i}\right)+S\left(x \mid D_{i}\right) \geq F_{i}\left(x, \tilde{p}_{i}\right)+x^{T} \tilde{p}_{i}, \forall \tilde{p}_{i} \in P_{i}
$$

and

$$
G_{i}\left(x, \tilde{q}_{i}\right)-S\left(x \mid E_{i}\right) \leq G_{i}\left(x, \tilde{q}_{i}\right)-x^{T} \tilde{q}_{i}, \forall \tilde{q}_{i} \in Q_{i},
$$

we have from (3.25) that

$$
\sum_{i=1}^{k} \tilde{u}_{i}\left[\left(F_{i}\left(x, \tilde{p}_{i}\right)+S\left(x \mid D_{i}\right)\right)-\lambda\left(G_{i}\left(x, \tilde{q}_{i}\right)-S\left(x \mid E_{i}\right)\right)\right] \geq 0 .
$$


Next, we prove that $\tilde{x}$ is a robust optimal solution of the problem (NUP). We claim that, for some $i^{0} \in\{1,2, \ldots, k\}$,

$$
\left[\left(F_{i^{0}}\left(x, \tilde{p}_{i^{0}}\right)+S\left(x \mid D_{i^{0}}\right)\right)-\lambda\left(G_{i^{0}}\left(x, \tilde{q}_{i^{0}}\right)-S\left(x \mid E_{i^{0}}\right)\right)\right] \geq 0 .
$$

To the contrary, we assume that

$$
\left[\left(F_{i}\left(x, \tilde{p}_{i}\right)+S\left(x \mid D_{i}\right)\right)-\lambda\left(G_{i}\left(x, \tilde{q}_{i}\right)-S\left(x \mid E_{i}\right)\right)\right]<0, \forall i=1,2, \ldots, k .
$$

Using $\tilde{u}_{i} \in \zeta_{+}^{k}$, we obtain

$$
\sum_{i=1}^{k} \tilde{u}_{i}\left[\left(F_{i}\left(x, \tilde{p}_{i}\right)+S\left(x \mid D_{i}\right)\right)-\lambda\left(G_{i}\left(x, \tilde{q}_{i}\right)-S\left(x \mid E_{i}\right)\right)\right]<0, i=1,2, \ldots, k,
$$

which is a contradiction to (3.26). Thus the claim holds and

$$
\max _{\substack{1 \leq i \leq k \\\left(p_{i}, q_{i}\right) \in P_{i} \times Q_{i}}} \frac{F_{i}\left(x, p_{i}\right)+S\left(x \mid D_{i}\right)}{G_{i}\left(x, q_{i}\right)-S\left(x \mid E_{i}\right)} \geq \lambda=\max _{\substack{1 \leq i \leq k \\\left(p_{i}, q_{i}\right) \in P_{i} \times Q_{i}}} \frac{F_{i}\left(\tilde{x}, \tilde{p}_{i}\right)+S\left(\tilde{x} \mid D_{i}\right)}{G_{i}\left(\tilde{x}, \tilde{q}_{i}\right)-S\left(\tilde{x} \mid E_{i}\right)}, \quad \forall x \in C .
$$

Hence, $\tilde{x}$ is a robust optimal solution of (NUP).

Theorem 3.2. Let $\tilde{x}$ be a feasible point of the problem (NUP). Then, the following statements are equivalent:

(A) at $\tilde{x} \in C$, the robust subdifferential constraint qualification (RSCQ) holds;

(B) $\tilde{x} \in C$ is a robust optimal solution of the problem (NUP) with optimal value $\lambda$ if and only if $\exists\left(\tilde{u}_{i}\right) \in \zeta_{+}^{k}, \tilde{p}_{i} \in P_{i}, \tilde{q}_{i} \in Q_{i}, \tilde{r}_{j} \in R_{j}$ and $\tilde{\gamma}_{j} \in K^{*}, i=1,2, \ldots, k, j=1,2, \ldots, m$ such that (3.1), (3.2) and (3.3) are satisfied.

Proof. From Theorem 3.1, we can conclude the proof $(i) \Rightarrow(i i)$ immediately. It remains to prove that $(i i) \Rightarrow(i)$. We only need to show

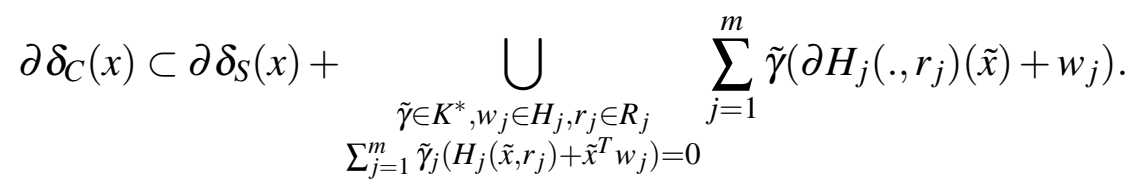

Letting $\tilde{y} \in \partial \delta_{C}(x)$, one has

$$
\langle\tilde{y}, x-\tilde{x}\rangle \leq 0, \forall x \in C
$$

For $i=1,2, \ldots, k$, one defines

$$
F_{i}\left(x, p_{i}\right)+S\left(x \mid D_{i}\right)=-\langle\tilde{y}, x\rangle \text { and } G_{i}\left(x, q_{i}\right)-S\left(x \mid E_{i}\right)=1 .
$$

From (3.27), one has $-\langle\tilde{y}, x\rangle \geq-\langle\tilde{y}, \tilde{x}\rangle$. Hence, for any $x \in C$,

$$
\max _{\substack{1 \leq i \leq k \\\left(p_{i}, q_{i}\right) \in P_{i} \times Q_{i}}} \frac{F_{i}\left(x, p_{i}\right)+S\left(x \mid D_{i}\right)}{G_{i}\left(x, q_{i}\right)-S\left(x \mid E_{i}\right)} \geq \max _{\substack{1 \leq i \leq k \\\left(p_{i}, q_{i}\right) \in P_{i} \times Q_{i}}} \frac{F_{i}\left(\tilde{x}, p_{i}\right)+S\left(\tilde{x} \mid D_{i}\right)}{G_{i}\left(\tilde{x}, q_{i}\right)-S\left(\tilde{x} \mid E_{i}\right)} .
$$

Consequently, $\tilde{x}$ is an optimal solution of (NUP). Also,

$$
\partial\left(F_{i}\left(x, p_{i}\right)+S\left(x \mid D_{i}\right)\right)=-\tilde{y}
$$

and

$$
\partial\left(G_{i}\left(x, q_{i}\right)-S\left(x \mid E_{i}\right)\right)=0
$$


From (3.1), we get

$$
\begin{gathered}
0 \in-\tilde{y}+\partial \delta_{C}(\tilde{x})+\sum_{j=1}^{m}\left[\tilde{\gamma}_{i}\left(\partial H_{j}\left(\tilde{x}, \tilde{r}_{j}\right)+w_{j}\right)\right] \\
\Rightarrow \tilde{y} \in \partial \delta_{C}(\tilde{x})+\sum_{j=1}^{m}\left[\tilde{\gamma}_{i}\left(\partial H_{j}\left(\tilde{x}, \tilde{r}_{j}\right)+w_{j}\right)\right] \text { and } \sum_{j=1}^{m}\left[\tilde{\gamma}_{j}\left(H_{j}\left(\tilde{x}, \tilde{r}_{j}\right)+\tilde{x}^{T} w_{j}\right)\right]=0 .
\end{gathered}
$$

This completes the proof.

\section{DuAlity Relations}

In this section, a Wolfe type nondifferentiable robust dual and a Mond-Weir type nondifferentiable robust dual are introduced for (NUP). Utilizing the necessary and sufficient conditions developed in Section 3, we focus on the duality relations between the Wolfe type nondifferentiable primal-dual pair and the Mond-Weir type nondifferentiable primal-dual pair.

4.1. Wolfe type robust dual. For the problem (NUP), the usual Wolfe type nondifferentiable dual problem is given as

$$
\begin{gathered}
\text { (WND) } \max _{\substack{(x, \lambda) \in R^{n} \times R_{+} \\
\gamma \in K^{*}, u_{i} \in \zeta_{+}^{k}}} \lambda \text { s.t. } \\
0 \in \sum_{i=1}^{k} u_{i}\left[\left(\partial F_{i}\left(., p_{i}\right)(x)+d_{i}\right)-\lambda\left(\partial G_{i}\left(., q_{i}\right)(x)-e_{i}\right)\right]+\partial \delta_{C}(x)+\sum_{j=1}^{m}\left[\gamma_{i}\left(\partial H_{j}\left(., r_{j}\right)(x)+w_{j}\right)\right], \\
\sum_{i=1}^{k} u_{i}\left[\left(F_{i}\left(x, p_{i}\right)+x^{T} d_{i}\right)-\lambda\left(G_{i}\left(x, q_{i}\right)-x^{T} e_{i}\right)\right]+\sum_{j=1}^{m}\left[\gamma_{j}\left(H_{j}\left(x, r_{j}\right)+x^{T} w_{j}\right)\right] \geq 0 .
\end{gathered}
$$

After maximizing over all possible, $p_{i} \in P_{i}, q_{i} \in Q_{i}, r_{j} \in R_{j}, i=1,2, \ldots, k, j=1,2, \ldots, m$, the optimistic Wolfe type robust nondifferentiable counterparts of (WRD) is given as:

$$
\begin{gathered}
\text { (WRD) } \max _{\substack{(x, \lambda) \in R^{n} \times R_{+} \\
\gamma \in K^{*}, u_{i} \in \zeta_{+}^{k} \\
\left(p_{i}, q_{i}, r_{j}\right) \in P_{i} \times Q_{i} \times R_{j}}} \lambda \text { s.t. } \\
0 \in \sum_{i=1}^{k} u_{i}\left[\left(\partial F_{i}\left(., p_{i}\right)(x)+d_{i}\right)-\lambda\left(\partial G_{i}\left(., q_{i}\right)(x)-e_{i}\right)\right]+\partial \delta_{C}(x)+\sum_{j=1}^{m}\left[\gamma_{i}\left(\partial H_{j}\left(., r_{j}\right)(x)+w_{j}\right)\right], \\
\sum_{i=1}^{k} u_{i}\left[\left(F_{i}\left(x, p_{i}\right)+x^{T} d_{i}\right)-\lambda\left(G_{i}\left(x, q_{i}\right)-x^{T} e_{i}\right)\right]+\sum_{j=1}^{m}\left[\gamma_{j}\left(H_{j}\left(x, r_{j}\right)+x^{T} w_{j}\right)\right] \geq 0 .
\end{gathered}
$$

Next, we will give the duality relations between the primal (NUP) and the nondifferentiable optimistic Wolfe dual (WRD).

Theorem 4.1. (Weak duality). Let $\tilde{x}$ be a feasible point of (NUP) and let $\left(\tilde{x}, \tilde{\lambda}, \tilde{\gamma}, \tilde{u}_{i}, \tilde{p}_{i}, \tilde{q}_{i}, \tilde{r}_{j}, \tilde{d}_{i}, \tilde{e}_{i}\right.$, $\left.\tilde{w}_{j}\right)$ be a feasible point of (WRD). Then,

$$
\max _{\substack{1 \leq i \leq k \\\left(p_{i}, q_{i}\right) \in P_{i} \times Q_{i}}} \frac{F_{i}\left(x, p_{i}\right)+S\left(x \mid D_{i}\right)}{G_{i}\left(x, q_{i}\right)-S\left(x \mid E_{i}\right)} \geq \tilde{\lambda} .
$$


Proof. Since $\left(\tilde{x}, \tilde{\lambda}, \tilde{\gamma}, \tilde{u}_{i}, \tilde{p}_{i}, \tilde{q}_{i}, \tilde{r}_{j}, \tilde{d}_{i}, \tilde{e}_{i}, \tilde{w}_{j}\right)$ is a feasible point of (WRD), we have

$$
0 \in \sum_{i=1}^{k} \tilde{u}_{i}\left[\left(\partial F_{i}\left(., \tilde{p}_{i}\right)(\tilde{x})+\tilde{d}_{i}\right)-\tilde{\lambda}\left(\partial G_{i}\left(., \tilde{q}_{i}\right)(\tilde{x})-\tilde{e}_{i}\right)\right]+\partial \delta_{C}(\tilde{x})+\sum_{j=1}^{m}\left[\tilde{\gamma}_{j}\left(\partial H_{j}\left(., \tilde{r}_{j}\right)+\tilde{w}_{j}\right)\right]
$$

and

$$
\sum_{i=1}^{k} \tilde{u}_{i}\left[\left(F_{i}\left(\tilde{x}, \tilde{p}_{i}\right)+\tilde{x}^{T} \tilde{d}_{i}\right)-\tilde{\lambda}\left(G_{i}\left(\tilde{x}, \tilde{q}_{i}\right)-\tilde{x}^{T} \tilde{e}_{i}\right)\right]+\sum_{j=1}^{m}\left[\tilde{\gamma}_{j}\left(H_{j}\left(\tilde{x}, \tilde{r}_{j}\right)+\tilde{x}^{T} \tilde{w}_{j}\right)\right] \geq 0
$$

From equation (4.1), it follows that there exist $\tilde{X}_{i} \in\left(\partial F_{i}\left(., \tilde{p}_{i}\right)(\tilde{x})-\tilde{\lambda} \partial G_{i}\left(., \tilde{q}_{i}\right)(\tilde{x})\right)$ and $\tilde{z} \in$ $\partial \delta_{C}(\tilde{x})$ such that

$$
-\sum_{i=1}^{k} \tilde{u}_{i} \tilde{X}_{i}-\sum_{i=1}^{k} \tilde{u}_{i}\left(\tilde{d}_{i}+\tilde{\lambda} \tilde{e}_{i}\right)-\tilde{z}-\sum_{j=1}^{m} \tilde{\gamma}_{j} \tilde{w}_{j} \in \sum_{j=1}^{m} \tilde{\gamma}_{j} \partial H_{j}\left(., \tilde{r}_{j}\right)(\tilde{x}) .
$$

Now, $\tilde{X}_{i} \in\left(\partial F_{i}\left(., \tilde{p}_{i}\right)(\tilde{x})-\tilde{\lambda} \partial G_{i}\left(., \tilde{q}_{i}\right)(\tilde{x})\right)$ and $\tilde{z} \in \partial \delta_{C}(\tilde{x})$ imply

$$
\begin{gathered}
\left(F_{i}\left(x, \tilde{p}_{i}\right)-\lambda G_{i}\left(x, \tilde{q}_{i}\right)\right)-\left(F_{i}\left(\tilde{x}, \tilde{p}_{i}\right)-\lambda G_{i}\left(\tilde{x}, \tilde{q}_{i}\right)\right) \geq\left\langle\tilde{X}_{i}, x-\tilde{x}\right\rangle, i=1,2, \ldots, k \\
\text { and } 0 \geq\langle\tilde{z}, x-\tilde{x}\rangle .
\end{gathered}
$$

It follows from (4.5) that

$$
\begin{aligned}
& \sum_{j=1}^{m} \tilde{\gamma}_{j} \partial H_{j}\left(x, \tilde{r}_{j}\right)-\sum_{j=1}^{m} \tilde{\gamma}_{j} \partial H_{j}\left(\tilde{x}, \tilde{r}_{j}\right) \\
& \geq\left\langle\left(-\sum_{i=1}^{k} \tilde{u}_{i} \tilde{X}_{i}-\sum_{i=1}^{k} \tilde{u}_{i}\left(\tilde{d}_{i}+\tilde{\lambda} \tilde{e}_{i}\right)-\tilde{z}-\sum_{j=1}^{m} \tilde{\gamma}_{j} \tilde{w}_{j}\right), x-\tilde{x}\right\rangle .
\end{aligned}
$$

Using (4.7) and (4.8), we arrive at

$$
\begin{aligned}
& \sum_{i=1}^{k} \tilde{u}_{i}\left[\left(F_{i}\left(x, \tilde{p}_{i}\right)-\lambda G_{i}\left(x, \tilde{q}_{i}\right)\right)-\left(F_{i}\left(\tilde{x}, \tilde{p}_{i}\right)-\lambda G_{i}\left(\tilde{x}, \tilde{q}_{i}\right)\right)\right]+\sum_{j=1}^{m} \tilde{\gamma}_{j} H_{j}\left(x, \tilde{r}_{j}\right)-\sum_{j=1}^{m} \tilde{\gamma}_{j} H_{j}\left(\tilde{x}, \tilde{r}_{j}\right) \\
& \geq-\left\langle\left(\sum_{i=1}^{k} \tilde{u}_{i}\left(\tilde{d}_{i}+\tilde{\lambda} \tilde{e}_{i}\right)+\sum_{j=1}^{m} \tilde{\gamma}_{j} \tilde{w}_{j}\right), x-\tilde{x}\right\rangle .
\end{aligned}
$$

By the dual constraint of (OWRD) in the above inequality, we obtain

$$
\sum_{i=1}^{k} \tilde{u}_{i}\left[\left(F_{i}\left(x, \tilde{p}_{i}\right)+x^{T} \tilde{d}_{i}\right)-\lambda\left(G_{i}\left(x, \tilde{q}_{i}\right)-x^{T} \tilde{e}_{i}\right)\right]+\sum_{j=1}^{m}\left[\tilde{\gamma}_{j}\left(H_{j}\left(x, \tilde{r}_{j}\right)+x^{T} \tilde{w}_{j}\right)\right] \geq 0 .
$$

From the facts that $S\left(x \mid W_{j}\right) \geq x^{T} w_{j}, \tilde{\gamma}_{j} \in K^{*}$, and $-\left\{H_{j}\left(x, \tilde{r}_{j}\right)+S\left(x \mid W_{j}\right)\right\} \in K$, we get from (4.9) that

$$
\sum_{i=1}^{k} \tilde{u}_{i}\left[\left(F_{i}\left(x, \tilde{p}_{i}\right)+x^{T} \tilde{d}_{i}\right)-\tilde{\lambda}\left(G_{i}\left(x, \tilde{q}_{i}\right)-x^{T} \tilde{e}_{i}\right)\right] \geq 0
$$


Since $F_{i}\left(x, \tilde{p}_{i}\right)+S\left(x \mid D_{i}\right) \geq F_{i}\left(x, \tilde{p}_{i}\right)+x^{T} \tilde{p}_{i}$ for all $\tilde{p}_{i} \in P_{i}$ and $G_{i}\left(x, \tilde{q}_{i}\right)-S\left(x \mid E_{i}\right) \leq G_{i}\left(x, \tilde{q}_{i}\right)-$ $x^{T} \tilde{q}_{i}$ for all $\tilde{q}_{i} \in Q_{i}$, we have from (4.10) that

$$
\sum_{i=1}^{k} \tilde{u}_{i}\left[\left(F_{i}\left(x, \tilde{p}_{i}\right)+S\left(x \mid D_{i}\right)\right)-\tilde{\lambda}\left(G_{i}\left(x, \tilde{q}_{i}\right)-S\left(x \mid E_{i}\right)\right)\right] \geq 0 .
$$

We claim that, for some $i^{0} \in\{1,2, \ldots, k\}$,

$$
\left[\left(F_{i^{0}}\left(x, \tilde{p}_{i^{0}}\right)+S\left(x \mid D_{i^{0}}\right)\right)-\tilde{\lambda}\left(G_{i^{0}}\left(x, \tilde{q}_{i^{0}}\right)-S\left(x \mid E_{i^{0}}\right)\right)\right] \geq 0 .
$$

To the contrary, assume that

$$
\left[\left(F_{i}\left(x, \tilde{p}_{i}\right)+S\left(x \mid D_{i}\right)\right)-\tilde{\lambda}\left(G_{i}\left(x, \tilde{q}_{i}\right)-S\left(x \mid E_{i}\right)\right)\right]<0, \text { for all } i=1,2, \ldots, k .
$$

Then it follows from $\tilde{u}_{i} \in \zeta_{+}^{k}$ and $\sum_{i=1}^{k} \tilde{u}_{i}=1$ that

$$
\sum_{i=1}^{k} \tilde{u}_{i}\left[\left(F_{i}\left(x, \tilde{p}_{i}\right)+S\left(x \mid D_{i}\right)\right)-\tilde{\lambda}\left(G_{i}\left(x, \tilde{q}_{i}\right)-S\left(x \mid E_{i}\right)\right)\right]<0, i=1,2, \ldots, k,
$$

which contradicts (4.8). Consequently,

$$
\max _{\substack{1 \leq i \leq k \\\left(p_{i}, q_{i}\right) \in P_{i} \times Q_{i}}} \frac{F_{i}\left(x, p_{i}\right)+S\left(x \mid D_{i}\right)}{G_{i}\left(x, q_{i}\right)-S\left(x \mid E_{i}\right)} \geq \tilde{\lambda} .
$$

The proof is complete.

Theorem 4.2. (Strong duality). Let $\tilde{x}$ be a feasible solution of (NUP). Then, the following are equivalent:

(A) at $\tilde{x} \in C$, the robust subdifferential constraint qualification (RSCQ) holds;

$(B)$ if $\tilde{x} \in C$ is a robust optimal solution of (NUP) with optimal value $\lambda$, then $\exists\left(\tilde{u}_{i}\right) \in$ $\zeta_{+}^{k}, \tilde{p}_{i} \in P_{i}, \tilde{q}_{i} \in Q_{i}, \tilde{r}_{j} \in R_{j}, \tilde{d}_{i} \in D_{i}, \tilde{e}_{i} \in E_{i}, \tilde{w}_{j} \in W_{j}$ and $\tilde{\gamma}_{j} \in K^{*}, i=1,2, \ldots, k, j=$ $1,2, \ldots, m$ such that $\left(\tilde{x}, \tilde{\lambda}, \tilde{\gamma}, \tilde{u}_{i}, \tilde{p}_{i}, \tilde{q}_{i}, \tilde{r}_{j}, \tilde{d}_{i}, \tilde{e}_{i}, \tilde{w}_{j}\right)$ is an optimal solution of (WRD), and the optimal values of the primal problem (NUP) and the dual problem (WRD) are equal.

Proof. $(i) \Rightarrow(i i)$. Let $\tilde{x} \in C$ be a robust optimal solution of (NUP) with optimal value $\tilde{\lambda}$. From Theorem 3.2, we see that there exit $\left(\tilde{u}_{i}\right) \in \zeta_{+}^{k}, \tilde{p}_{i} \in P_{i}, \tilde{q}_{i} \in Q_{i}, \tilde{r}_{j} \in R_{j}$ and $\tilde{\gamma}_{j} \in K^{*}, i=$ $1,2, \ldots, k, j=1,2, \ldots, m$ such that (3.1), (3.2) and (3.3) hold. Then $\left(\tilde{x}, \tilde{\lambda}, \tilde{\gamma}, \tilde{u}_{i}, \tilde{p}_{i}, \tilde{q}_{i}, \tilde{r}_{j}, \tilde{d}_{i}, \tilde{e}_{i}, \tilde{w}_{j}\right)$ is a feasible solution of (WRD). Consequently, $\tilde{\lambda}$ is less than or equal to optimal value of the problem (WRD). But, from Theorem 4.1, we see that the optimal value of (NUP) is greater than or equal to the optimal value of the (WRD). Therefore, the optimal value of the primal (NUP) is equal to the optimal value of the dual problem (WRD).

From the proof of Theorem 3.2, we conclude $(i i) \Rightarrow(i)$ easily.

4.2. Mond-Weir type robust dual. For the primal (NUP), the conventional Mond-Weir type nondifferentiable dual problem is given as:

$$
\text { (MND) } \max _{\substack{(x, \lambda) \in R^{n} \times R_{+} \\ \gamma \in K^{*}, u_{i} \in \zeta_{+}^{k}}} \lambda \text { s.t. }
$$




$$
\begin{gathered}
0 \in \sum_{i=1}^{k} u_{i}\left[\left(\partial F_{i}\left(., p_{i}\right)(x)+d_{i}\right)-\lambda\left(\partial G_{i}\left(., q_{i}\right)(x)-e_{i}\right)\right]+\partial \delta_{C}(x)+\sum_{j=1}^{m}\left[\gamma_{j}\left(\partial H_{j}\left(., r_{j}\right)(x)+w_{j}\right)\right] \\
\sum_{i=1}^{k} u_{i}\left[\left(F_{i}\left(x, p_{i}\right)+x^{T} d_{i}\right)-\lambda\left(G_{i}\left(x, q_{i}\right)-x^{T} e_{i}\right)\right] \geq 0 \\
\sum_{j=1}^{m}\left[\gamma_{j}\left(H_{j}\left(x, r_{j}\right)+x^{T} w_{j}\right)\right] \geq 0 .
\end{gathered}
$$

After maximizing over all possible, $p_{i} \in P_{i}, q_{i} \in Q_{i}, w_{j} \in W_{j}$, the optimistic Mond-Weir type robust nondifferentiable counterparts of (MRD) is given below:

$$
\begin{gathered}
\text { (MRD) } \max _{\substack{(x, \lambda) \in R^{n} \times R_{+} \\
\gamma \in K^{*}, u_{i} \in \zeta_{+}^{k} \\
\left(p_{i}, q_{i}, r_{j}\right) \in P_{i} \times Q_{i} \times R_{j}}}^{\lambda \text { s.t. }} \\
0 \in \sum_{i=1}^{k} u_{i}\left[\left(\partial F_{i}\left(., p_{i}\right)(x)+d_{i}\right)-\lambda\left(\partial G_{i}\left(., q_{i}\right)(x)-e_{i}\right)\right]+\partial \delta_{C}(x)+\sum_{j=1}^{m}\left[\gamma_{i}\left(\partial H_{j}\left(., r_{j}\right)(x)+w_{j}\right)\right], \\
\sum_{i=1}^{k} u_{i}\left[\left(F_{i}\left(x, p_{i}\right)+x^{T} d_{i}\right)-\lambda\left(G_{i}\left(x, q_{i}\right)-x^{T} e_{i}\right)\right] \geq 0, \\
\sum_{j=1}^{m}\left[\gamma_{j}\left(H_{j}\left(x, r_{j}\right)+x^{T} w_{j}\right)\right] \geq 0 .
\end{gathered}
$$

In the following, we give the duality relations between the primal (NUP) and the nondifferentiable optimistic Mond-Weir dual (MRD).

Theorem 4.3. (Weak duality). Let $\tilde{x}$ be a feasible point of (NUP) and let $\left(\tilde{x}, \tilde{\lambda}, \tilde{\gamma}, \tilde{u}_{i}, \tilde{p}_{i}, \tilde{q}_{i}, \tilde{r}_{j}, \tilde{d}_{i}, \tilde{e}_{i}\right.$, $\left.\tilde{w}_{j}\right)$ be a feasible point of (MRD). Then,

$$
\max _{\substack{1 \leq i \leq k \\\left(p_{i}, q_{i}\right) \in P_{i} \times Q_{i}}} \frac{F_{i}\left(x, p_{i}\right)+S\left(x \mid D_{i}\right)}{G_{i}\left(x, q_{i}\right)-S\left(x \mid E_{i}\right)} \geq \tilde{\lambda}
$$

Proof. Since $\left(\tilde{x}, \tilde{\lambda}, \tilde{\gamma}, \tilde{u}_{i}, \tilde{p}_{i}, \tilde{q}_{i}, \tilde{r}_{j}, \tilde{d}_{i}, \tilde{e}_{i}, \tilde{w}_{j}\right)$ is a feasible point of (MRD), we find from (4.14) that there exist $\tilde{X}_{i} \in\left(\partial F_{i}\left(., \tilde{p}_{i}\right)(\tilde{x})-\tilde{\lambda} \partial G_{i}\left(., \tilde{q}_{i}\right)(\tilde{x})\right)$ and $\tilde{z} \in \partial \delta_{C}(\tilde{x})$ such that

$$
-\sum_{i=1}^{k} \tilde{u}_{i} \tilde{X}_{i}-\sum_{i=1}^{k} \tilde{u}_{i}\left(\tilde{d}_{i}+\tilde{\lambda} \tilde{e}_{i}\right)-\tilde{z}-\sum_{j=1}^{m} \tilde{\gamma}_{j} \tilde{w}_{j} \in \sum_{j=1}^{m} \tilde{\gamma}_{j} \partial H_{j}\left(., \tilde{r}_{j}\right)(\tilde{x}) .
$$

From $\tilde{X}_{i} \in\left(\partial F_{i}\left(., \tilde{p}_{i}\right)(\tilde{x})-\tilde{\lambda} \partial G_{i}\left(., \tilde{q}_{i}\right)(\tilde{x})\right)$ and $\tilde{z} \in \partial \delta_{C}(\tilde{x})$, we have

$$
\begin{gathered}
\left(F_{i}\left(x, \tilde{p}_{i}\right)-\lambda G_{i}\left(x, \tilde{q}_{i}\right)\right)-\left(F_{i}\left(\tilde{x}, \tilde{p}_{i}\right)-\lambda G_{i}\left(\tilde{x}, \tilde{q}_{i}\right)\right) \geq\left\langle\tilde{X}_{i}, x-\tilde{x}\right\rangle, i=1,2, \ldots, k \\
\text { and } 0 \geq\langle\tilde{z}, x-\tilde{x}\rangle .
\end{gathered}
$$

The expression (4.15) implies

$$
\sum_{j=1}^{m} \tilde{\gamma}_{j} \partial H_{j}\left(x, \tilde{r}_{j}\right)-\sum_{j=1}^{m} \tilde{\gamma}_{j} \partial H_{j}\left(\tilde{x}, \tilde{r}_{j}\right) \geq\left\langle\left(-\sum_{i=1}^{k} \tilde{u}_{i} \tilde{X}_{i}-\sum_{i=1}^{k} \tilde{u}_{i}\left(\tilde{d}_{i}+\tilde{\lambda} \tilde{e}_{i}\right)-\tilde{z}-\sum_{j=1}^{m} \tilde{\gamma}_{j} \tilde{w}_{j}\right), x-\tilde{x}\right\rangle .
$$


It follows that

$$
\begin{aligned}
& \sum_{i=1}^{k} \tilde{u}_{i}\left[\left(F_{i}\left(x, \tilde{p}_{i}\right)-\lambda G_{i}\left(x, \tilde{q}_{i}\right)\right)-\left(F_{i}\left(\tilde{x}, \tilde{p}_{i}\right)-\lambda G_{i}\left(\tilde{x}, \tilde{q}_{i}\right)\right)\right]+\sum_{j=1}^{m} \tilde{\gamma}_{j} H_{j}\left(x, \tilde{r}_{j}\right)-\sum_{j=1}^{m} \tilde{\gamma}_{j} H_{j}\left(\tilde{x}, \tilde{r}_{j}\right) \\
& \geq-\left\langle\left(\sum_{i=1}^{k} \tilde{u}_{i}\left(\tilde{d}_{i}+\tilde{\lambda} \tilde{e}_{i}\right)+\sum_{j=1}^{m} \tilde{\gamma}_{j} \tilde{w}_{j}\right), x-\tilde{x}\right\rangle .
\end{aligned}
$$

Using the second and the third constraints of (MRD) in the above inequality, we obtain

$$
\sum_{i=1}^{k} \tilde{u}_{i}\left[\left(F_{i}\left(x, \tilde{p}_{i}\right)+x^{T} d_{i}\right)-\lambda\left(G_{i}\left(x, \tilde{q}_{i}\right)-x^{T} e_{i}\right)\right]+\sum_{j=1}^{m}\left[\tilde{\gamma}_{j}\left(H_{j}\left(x, \tilde{r}_{j}\right)+x^{T} w_{j}\right)\right] \geq 0 .
$$

The rest of the proof follows along the lines of the Theorem 4.1.

Theorem 4.4. (Strong duality). Suppose that $\tilde{x}$ is a feasible solution for the problem (NUP). Then, the following are equivalent:

(A) at $\tilde{x} \in C$, the robust subdifferential constraint qualification (RSCQ) holds;

$(B)$ if $\tilde{x} \in C$ is a robust optimal solution of the problem (NUP) with optimal value $\lambda$, then there exist $\left(\tilde{u}_{i}\right) \in \zeta_{+}^{k}, \tilde{p}_{i} \in P_{i}, \tilde{q}_{i} \in Q_{i}, \tilde{r}_{j} \in R_{j}, \tilde{d}_{i} \in D_{i}, \tilde{e}_{i} \in E_{i}, \tilde{w}_{j} \in W_{j}$ and $\tilde{\gamma}_{j} \in K^{*}, i=$ $1,2, \ldots, k, j=1,2, \ldots, m$ such that $\left(\tilde{x}, \tilde{\lambda}, \tilde{\gamma}, \tilde{u}_{i}, \tilde{p}_{i}, \tilde{q}_{i}, \tilde{r}_{j}, \tilde{d}_{i}, \tilde{e}_{i}, \tilde{w}_{j}\right)$ is an optimal solution of $(M R D)$, and the optimal value of (NUP) equals to the optimal value of (MRD).

Proof. The proof is similar to that of Theorem 4.2. So, we omit here.

\section{Robustness in Quadratic Fractional Programming Problems}

In (NUP), let $S=R^{n}$ and $K=R_{+}^{t}$ and consider $S\left(x \mid D_{i}\right)=S\left(x \mid E_{i}\right)=S\left(x \mid W_{j}\right)=0, i=1,2, \ldots, k$, $j=1,2, \ldots, m$. Then the uncertain quadratic minimax fractional problem is given as

$$
\text { (UQFP) } \min _{x \in R^{n}} \max _{1 \leq i \leq k} \frac{\frac{1}{2} x^{T} C_{i} x+r_{i}^{T} x+s_{i}}{\frac{1}{2} x^{T} D_{i} x+u_{i}^{T} x+v_{i}}
$$

s.t.

$$
a_{\tau}^{T} x \leq b_{\tau}, \tau=1,2, \ldots, t
$$

where, for $i=1,2, \ldots, k$,

(i) $C_{i}$ is a $n \times n$ positive definite matrix, $D_{i}$ is a $n \times n$ negative semidefinite matrix and $r_{i}, u_{i} \in$ $R^{n}$. Assume that $\frac{1}{2} x^{T} C_{i} x+r_{i}^{T} x+s_{i} \geq 0$ for some $x \in R^{n}$ and $\frac{1}{2} x^{T} D_{i} x+u_{i}^{T} x+v_{i}>0$ for all $x \in R^{n}$;

(ii) $p_{i}:=\left(C_{i}, r_{i}, s_{i}\right) \in P_{i}, q_{i}:=\left(D_{i}, u_{i}, v_{i}\right) \in Q_{i}$ and $w_{\tau}:=\left(a_{\tau}, b_{\tau}\right) \in W_{\tau}, P_{i}, Q_{i}, W_{\tau}$ represents the uncertain compact, convex data sets in $R^{n \times n} \times R^{n} \times R$.

We assume that the feasible set of (UQFP) is non-empty. The problem (UQFP) can be written as:

$$
\min _{x \in R^{n}} \max _{1 \leq i \leq k} \frac{1}{2} x^{T} K(\xi) x+c(\xi)^{T} x+d(\xi)
$$

s.t. $a_{\tau}^{T} x \leq b_{\tau}, \tau=1,2, \ldots, t$, where $K(\xi)=C_{i}-\xi D_{i}$ is positive definite for $\xi \geq 0, c(\xi)=r_{i}-\xi u_{i}$ and $d(\xi)=s_{i}-\xi v_{i}$. 
Now, the robust counterpart of the problem (UQFP) can be constructed as follows:

$$
\text { (RQFP) } \min _{x \in R^{n}} \max _{1 \leq i \leq k} \max _{\substack{\left(C_{i}, r_{i}, s_{i}\right) \in P_{i} \\\left(D_{i}, u_{i}, v_{i}\right) \in Q_{i}}} \frac{1}{2} x^{T} K(\xi) x+c(\xi)^{T} x+d(\xi)
$$

s.t.

$$
\begin{gathered}
a_{\tau}^{T} x \leq b_{\tau}, \tau=1,2, \ldots, t, \\
\left(a_{\tau}, b_{\tau}\right) \in W_{\tau}, \tau=1,2, \ldots, t .
\end{gathered}
$$

In the following discussion, the Wolfe robust dual of the problem (RQFP) is constructed under uncertainty in the objective functions and the constraints.

5.1. Scenario uncertainty in quadratic fractional minimax problem. Consider the problem (RQFP) under scenario uncertainty in the objectives and the constraint data sets. The objective data sets are given by:

$$
P_{i}=\operatorname{co}\left\{\left(C_{i 1}, r_{i 1}, s_{i 1}\right), \ldots,\left(C_{i J}, r_{i J}, s_{i J}\right)\right\}
$$

and

$$
Q_{i}=\operatorname{co}\left\{\left(D_{i 1}, u_{i 1}, v_{i 1}\right), \ldots,\left(D_{i L}, u_{i L}, v_{i L}\right)\right\},
$$

where $\left(C_{i j}, r_{i j}, s_{i j}\right),\left(D_{i l}, u_{i l}, v_{i l}\right) \in R^{n \times n} \times R^{n} \times R, i=1,2, \ldots, k, j=1,2, \ldots, J, l=1,2, \ldots, L$. The constraint data set is given by:

$$
W_{\tau}=\operatorname{co}\left\{\left(a_{\tau 1}, b_{\tau 1}\right), \ldots,\left(a_{\tau T}, b_{\tau T}\right)\right\},
$$

where $\left(a_{\tau m}, b_{\tau m}\right) \in R^{n} \times R, \tau=1,2, \ldots, t, m=1,2, \ldots, T$. With the above data sets, the optimistic Wolfe counterpart of (RQFP) can be written as:

$$
\begin{gathered}
\max _{\substack{(x, \lambda) \in R^{n} \times R \\
\rho_{i} \in \zeta_{+}^{k}, \gamma \in R_{+}^{t}}} \lambda \text { s.t. } \\
\sum_{i=1}^{k} \rho_{i}\left\{\left(C_{i} x+r_{i}\right)-\xi\left(D_{i} x+u_{i}\right)\right\}+\sum_{\tau=1}^{t} \gamma_{\tau} a_{\tau}=0, \\
\sum_{i=1}^{k} \rho_{i}\left\{\left(\frac{1}{2} x^{T} C_{i} x+r_{i}^{T} x+s_{i}\right)-\xi\left(\frac{1}{2} x^{T} D_{i} x+u_{i}^{T} x+v_{i}\right)\right\}+\sum_{\tau=1}^{t} \gamma_{\tau}\left(a_{\tau}^{T} x-b_{\tau}\right) \geq 0, \\
\left(C_{i}, r_{i}, s_{i}\right)=\sum_{j=1}^{J} \eta_{i j}\left(C_{i j}, r_{i j}, s_{i j}\right), \sum_{j=1}^{J} \eta_{i j}=1, \eta_{i j} \geq 0, \forall j, \\
\left(D_{i}, u_{i}, v_{i}\right)=\sum_{l=1}^{L} \mu_{i l}\left(D_{i l}, u_{i l}, v_{i l}\right), \sum_{l=1}^{L} \mu_{i l}=1, \mu_{i l} \geq 0, \forall l, \\
\left(a_{\tau}, b_{\tau}\right)=\sum_{m=1}^{T} v_{\tau m}\left(a_{\tau m}, b_{\tau m}\right), \sum_{m=1}^{T} v_{\tau m}=1, v_{\tau m} \geq 0, \forall m .
\end{gathered}
$$

From (5.1), (5.2) and (5.3), we obtain

$$
\left(C_{i}, r_{i}, s_{i}\right)=\sum_{j=1}^{J} \sum_{l=1}^{L} \eta_{i j} \mu_{i l}\left(C_{i j}, r_{i j}, s_{i j}\right),\left(D_{i}, u_{i}, v_{i}\right)=\sum_{j=1}^{J} \sum_{l=1}^{L} \eta_{i j} \mu_{i l}\left(D_{i l}, u_{i l}, v_{i l}\right) .
$$


Taking $\rho_{i j l}=\rho_{i} \eta_{i j} \mu_{i l}$ and $\gamma_{\tau m}=\gamma_{\tau} \nu_{\tau m}$, it follows that $\rho_{i j l} \in \zeta_{+}^{k J L}$ and $\gamma_{\tau m} \in R_{+}^{t T}$. Therefore, the optimistic counterpart of the Wolfe type dual of (RQFP) is converted to:

$$
\begin{aligned}
& \text { (OQFP) } \max _{(x, \lambda) \in R^{n} \times R_{+}} \lambda \text { s.t. } \\
& \rho_{i j l} \in \zeta_{+}^{k J L, \gamma \tau m \in R_{+}^{t T}} \\
& \sum_{i=1}^{k} \sum_{j=1}^{J} \sum_{l=1}^{L} \rho_{i j l}\left\{\left(C_{i j} x+r_{i j}\right)-\lambda\left(D_{i l} x+u_{i l}\right)\right\}+\sum_{\tau=1}^{t} \sum_{m=1}^{T} \gamma_{\tau m} a_{\tau m}=0, \\
& \sum_{i=1}^{k} \sum_{j=1}^{J} \sum_{l=1}^{L} \rho_{i j l}\left\{\left(s_{i j}-\lambda v_{i l}\right)\right\}+\sum_{\tau=1}^{t} \sum_{m=1}^{T} \gamma_{\tau m} b_{\tau m} \geq 0,
\end{aligned}
$$

which is a linear programming problem. Thus the dual problem (OQFP) can be solved easily using computational softwares and the solutions can be obtained.

Remark 5.1. If the positive definite matrices $C_{i}$ and the negative semidefinite matrices $D_{i}$, $i=1,2, \ldots, k$ are considered to be null matrices, then the problem (UQFP) is reduced to the problem (UFLP) in [28] and the problem (OQFP) becomes the problem (ODFLP) ${ }_{W}^{1}$ in [28].

Next, we establish a relation between the problem (RQFP) and (OQFP).

Theorem 5.2. (Strong duality). Suppose that $\tilde{x}$ is an optimal solution of the problem (RQFP) with value $\lambda \in R$. Then, $\exists \rho=\rho_{i j l} \in \zeta_{+}^{k J L}$ and $\gamma=\gamma_{\tau m} \in R_{+}^{t T}$ such that $(\lambda, \rho, \gamma) \in R \times \zeta_{+}^{k J L} \times R_{+}^{t T}$ is an optimal solution of (OQFP) and optimal values of both the problems are same.

Proof. We define the functions $F_{i}\left(x, p_{i}\right), G_{i}\left(x, q_{i}\right)$ and $H_{\tau}\left(x, r_{\tau}\right), i=1,2, \ldots, k$ by

$$
F_{i}\left(x, p_{i}\right)=\frac{1}{2} x^{T} C_{i} x+r_{i}^{T} x+s_{i}, G_{i}\left(x, q_{i}\right)=\frac{1}{2} x^{T} D_{i} x+u_{i}^{T} x+v_{i}
$$

and

$$
H_{\tau}\left(x, r_{\tau}\right)=a_{\tau}^{T} x-b_{\tau}, \tau=1,2, \ldots, t .
$$

Consider $H(x, r)=\left(H_{\tau}\left(x, r_{\tau}\right)\right)$ and let $R=\prod_{\tau=1}^{t} R_{\tau}, r_{\tau} \in R_{\tau}$. Clearly, $H(., r)$ is a differentiable convex function. It is observed that all the hypothesis of Theorem 3.2 are satisfied. To show the strong duality relation between (RQFP) and (OQFP), it is sufficient to show that the (RSCQ) holds at $\tilde{x} \in C$. Hence, from Definition 2.6, we only need to show that

$$
\partial \delta_{C}(x) \subset \bigcup_{\substack{\tilde{\gamma} \in K^{*}, r_{j} \in R_{j} \\ \sum_{j=1}^{m} \tilde{\gamma}_{j} H_{j}\left(\tilde{x}, r_{j}\right)}} \partial \sum_{j=1}^{m} \tilde{\gamma} H_{j}\left(., r_{j}\right)(\tilde{x}),
$$

as $S\left(x \mid W_{j}\right)=0$ in (RQFP) and $\partial \delta_{C}(\tilde{x})=\partial \delta_{R^{n}}(\tilde{x})=\{0\}$. Let $\tilde{y} \in \partial \delta_{C}(\tilde{x})$. Then,

$$
\begin{aligned}
\delta_{C}(x)-\delta_{C}(\tilde{x}) & \geq\langle\tilde{y}, x-\tilde{x}\rangle \\
\Rightarrow\langle-\tilde{y}, x\rangle & \geq\langle-\tilde{y}, \tilde{x}\rangle, \forall x \in C .
\end{aligned}
$$

This implies

$$
\left\{x: a_{\tau}^{T} x \leq b_{\tau}, \forall \tau=1,2, \ldots, t,\right\} \subset\{x:\langle-\tilde{y}, x\rangle \geq\langle-\tilde{y}, \tilde{x}\rangle\},
$$

which further yields

$$
\left\{x: a_{\tau m}^{T} x \leq b_{\tau m}, \forall \tau=1,2, \ldots, t, m=1,2, \ldots, T\right\} \subset\{x:\langle-\tilde{y}, x\rangle \geq\langle-\tilde{y}, \tilde{x}\rangle\} .
$$


Using the Proposition 2.1 in [32], there exits $\gamma_{\tau m} \in R_{+}^{t T}$ such that

$$
-\tilde{y}+\sum_{\tau=1}^{t} \sum_{m=1}^{T} \gamma_{\tau m} a_{\tau m}=0,\langle-\tilde{y}, \tilde{x}\rangle+\sum_{\tau=1}^{t} \sum_{m=1}^{T} \gamma_{\tau m} b_{\tau m} \leq 0
$$

which is equivalent to

$$
\tilde{y}=\sum_{\tau=1}^{t} \sum_{m=1}^{T} \gamma_{\tau m} a_{\tau m}, \sum_{\tau=1}^{t} \sum_{m=1}^{T} \gamma_{\tau m} b_{\tau m} \leq \sum_{\tau=1}^{t} \sum_{m=1}^{T} \gamma_{\tau m} a_{\tau m} \tilde{x}
$$

From the fact that $\gamma_{\tau m} \in R_{+}^{t T}$ and the feasibility of (RQFP) at $\tilde{x}$, we obtain

$$
\tilde{y}=\sum_{\tau=1}^{t} \sum_{m=1}^{T} \gamma_{\tau m} a_{\tau m}, \sum_{\tau=1}^{t} \sum_{m=1}^{T} \gamma_{\tau m}\left(a_{\tau m}^{T} \tilde{x}-b_{\tau m}\right)=0 .
$$

Letting $\gamma_{\tau}=\sum_{m=1}^{T} \gamma_{\tau m} \geq 0, \tau=1,2, \ldots, t$, and for any $\left(\tilde{a}_{\tau}, \tilde{b}_{\tau}\right) \in W$, we define

$$
a_{\tau}= \begin{cases}\sum_{m=1}^{T} \frac{\gamma_{\tau m}}{\gamma_{\tau}} a_{\tau m}, & \text { if } \gamma_{\tau}>0 \\ \tilde{a}_{\tau m}, & \text { if } \gamma_{\tau}=0\end{cases}
$$

and

$$
b_{\tau}= \begin{cases}\sum_{m=1}^{T} \frac{\gamma_{\tau m}}{\gamma_{\tau}} b_{\tau m}, & \text { if } \gamma_{\tau}>0, \\ \tilde{b}_{\tau m}, & \text { if } \gamma_{\tau}=0,\end{cases}
$$

From the above definitions, it is clear that $\left(a_{\tau}, b_{\tau}\right) \in W$ as $W$ is a convex set. Therefore,

$$
\gamma_{\tau}\left(a_{\tau}, b_{\tau}\right)=\sum_{m=1}^{T} \gamma_{\tau m}\left(a_{\tau m}, b_{\tau m}\right)
$$

Substituting (5.5) into (5.4), we get

$$
\tilde{y}=\sum_{\tau=1}^{t} \gamma_{\tau} a_{\tau} \text { and } \sum_{\tau=1}^{t} \gamma_{\tau}\left(a_{\tau}^{T} \tilde{x}-b_{k}\right)=0
$$

Consequently,

$$
\tilde{y} \in \bigcup_{\substack{\tilde{\gamma} \in R_{+}^{t}, r_{j} \in R_{j} \\ \sum_{j=1}^{m} \tilde{\gamma}_{j} H_{j}\left(\tilde{x}, r_{j}\right)}} \partial \sum_{j=1}^{m} \tilde{\gamma} H_{j}\left(., r_{j}\right)(\tilde{x}) .
$$

This completes the proof.

\section{Acknowledgments}

This paper was supported by the Feng Tay project of National Yunlin University of Science and Technology, Taiwan. The authors are grateful to the referees for useful suggestions which improved this paper. 


\section{REFERENCES}

[1] S. Chandra, B.D. Craven, B. Mond, Generalized fractional programming duality: a ratio game approach, J. Aust. Math. Soc. Series B 28 (1986), 170-180.

[2] A. Cambini, E. Castagnoli, L. Martein, L.P. Mazzoleni, S. Schaible (Eds.), Generalized convexity and fractional programming with economic applications, In: Proceedings of the International Workshop on "Generalized Concavity, Fractional Programming and Economic Applications" Held at the University of Pisa, Italy, May 30 - June 1, 1988.

[3] J. Neuman, A model of general economic equilibrium, Rev. Econom. Stud. 13 (1945), 1-9.

[4] I.M. Stancu-Minasian, S. Tigan, On some fractional programming models occurring in minimum-risk problems, In: Cambini A., Castagnoli E., Martein L., Mazzoleni P., Schaible S. (eds) Generalized Convexity and Fractional Programming with Economic Applications. Lecture Notes in Economics and Mathematical Systems, vol 345. Springer, Berlin, Heidelberg, 1990.

[5] G.J. Zalmai, Optimality criteria and duality models for generalized fractional programming problems containing locally subdifferentiable and $\rho$-convex functions, Optimization, 32 (1995), 95-125.

[6] T. Antczak, Generalized fractional minimax programming with $B-(p, r)$-invexity, Comput. Math. Appl. 56 (2008), 1505-1525.

[7] I. Ahmad, Z. Husain, Optimality conditions and duality in nondifferentiable minimax fractional programming with generalized convexity, J. Optim. Theory Appl. 129 (2006), 255-275.

[8] S.K. Gupta, D. Dangar, I. Ahamd, On second order duality for nondifferentiable minimax fractional programming problems involving type-I functions, ANZIAM J. 55 (2014), 479-494.

[9] T. Antczak, Optimality and duality for minimax fractional programming with support functions under $B$ ( $p, r)$-Type I assumptions, Math. Comput. Modelling, 57 (2013) 1083-1100.

[10] M.A. Khan, F.R. Al-Solamy, Sufficiency and duality in nondifferentiable minimax fractional programming with $\left(H_{p}, r\right)$-invexity, J. Egyptian Math. Soc. 23 (2015) 208-213.

[11] E.K. Kervin, H.L.M. Kerivin and M. M. Wiecek, Robust multiobjective optimization problem with application to internet routing, Ann. Oper. Res. 271 (2018), 487-525.

[12] A. A. Khan, C. Tammer, C. Zalinescu, Set-Valued Optimization: An Introduction with Applications, Springer, Berlin, 2015.

[13] A. Ben-Tal, L.E. Ghaoui, A. Nemirovski, Robust Optimization, Princeton Series in Applied Mathematics, 2009.

[14] D. Bertsimas, D.S. Brown, C. Caramanis, Theory and applications of robust optimization, SIAM Rev. 53 (2011), 464-501.

[15] K. Deb, H. Gupta, Introducing robustness in multiobjective optimization, Evol. Comput. 14 (2006), 463-494.

[16] S. Gunawan, S. Azarm, Multiobjective robust optimization using a sensitivity region concept, Struct. Multidiscip. Optim. 29 (2005), 50-60.

[17] D. Kuroiwa, G.M. Lee, On robust multiobjective optimization, Vietnam J. Math. 40 (2012), 305-317.

[18] B.L. Gorissen, Robust fractional programming, J. Optim. Theory Appl. 166 (2015), 508-528.

[19] J.H. Lee, L.G. Jiao, Solving fractional multicriteria optimization problems with sum of squares convex polynomial data, J. Optim. Theory Appl. 176 (2018), 428-455.

[20] L. Jiao, J.H. Lee, Finding efficient solutions in robust multiple objective optimization with SOS-convex polynomial data, Ann. Oper. Res. 296 (2021), 803-820.

[21] V. Jeyakumar, G.Y. Li, Robust duality for fractional programming problems with constraint-wise data uncertainty, J. Optim. Theory Appl. 151 (2011), 292-303.

[22] X.K. Sun, Y. Chai, On robust duality for fractional programming with uncertainty data, Positivity 18 (2014), 9-28.

[23] M. A.Goberna, V. Jeyakumar, G. Li, J. Vicentre-Perez, Robust solutions to multi-objective linear programs with uncertain data, Eur. J. Oper. Res. 242 (2015), 730-743.

[24] T.D. Chuong, Optimality and duality for robustmultiobjective optimization problems, Nonlinear Anal .134 (2016), 127-143.

[25] J.H. Lee, G.M. Lee, On optimality conditions and duality theorems for robust semi-infinite multiobjective optimization problems, Ann. Oper. Res. 269 (2018), 419-438. 
[26] R. Bokrantz, A. Fredriksson, Necessary and sufficient conditions for Pareto efficiency in robust multiobjective optimization, Eur. J. Oper. Res. 262 (2017), 682-692.

[27] X.-K. Sun, X.-J. Long, H.-Y. Fu, X.-B. Li, Some characterizations of robust optimal solutions for uncertain fractional optimization and applications, J. Indust. Manage. Optim. 13 (2017), 803-824.

[28] X.-B. Li, Q.-L. Wang, Z. Lin, Optimality conditions and duality for minimax fractional programming problems with data uncertainty, J. Indust. Manage. Optim. 15 (2019), 1133-1151.

[29] W. Dinkelbach, On nonlinear fractional programming, Management Sci. 13 (1967), 492-498.

[30] I.P. Debnath, S.K. Gupta, Efficiency and duality for a vector of quotients of curvilinear functionals on the first-order jet bundle, Optim. Control Appl. Methods 38 (2017), 1227-1238.

[31] J.B. Hiriart-Urruty, C. Lemarechal, Convex Analysis and Minimization Algorithms I, Springer, Berlin, 1993.

[32] O.L. Mangasarian, Set containment characterization, J. Global Optim. 24 (2002), 473-480. 